\title{
Efficient Delegation by an Informed Principal ${ }^{1}$
}

\author{
By Eric W. Bond ${ }^{2}$ and Thomas A. Gresik ${ }^{3}$ \\ July 2010 \\ Forthcoming, Journal of Economics and Management Strategy
}

\begin{abstract}
Motivated by examples from the insurance industry, the automobile industry, retailing, and multinational strategy, we study an organizational structure we refer to as "partial delegation." In a bargaining problem between an informed party and an uninformed party, partial delegation involves the informed party delegating control of bargaining responsibilities to an agent while retaining control of its private information. We show that partial delegation, with or without an observable agency contract, enables the informed party to earn information rents without creating quantity distortions. That is, firstbest quantities are traded in equilibrium. In general, we argue that partial delegation allows an informed principal to promote more efficient agreements with outside parties while also endogenously improving its bargaining power.
\end{abstract}

JEL Numbers: C78, D82, L24

Keywords: Delegation, efficient bargaining, contracting

${ }^{1}$ We thank Thomas Cosimano, Matthew Mitchell, the referees and associate editor of this journal, and participants at the University of Iowa department seminar series for their comments. We thank Gregory Gundlach for introducing us to the practice of category management.

${ }^{2}$ Department of Economics, Vanderbilt University, VU Station B \#351819, 2301 Vanderbilt Place, Nashville, TN 37235-1819.

${ }^{3}$ Corresponding Author: Department of Economics, University of Notre Dame, Notre Dame, IN 46556.tgresik@nd.edu 


\section{Introduction.}

We examine a bargaining game in which an informed seller hires an agent to bargain with an uninformed buyer. It is well known that if the seller and buyer bargain directly over the price and quantity of the good, the outcome may be second best due to a misalignment of private information and bargaining power. For example, a buyer that has sufficient bargaining power will trade less than the first best quantity in order to limit the information rent to the informed seller. The question we address in this paper is whether these distortions could be reduced if the seller were to delegate bargaining to an agent. ${ }^{4}$

In a complete information setting, it has been well established that delegation can create a benefit by separating ownership from control. ${ }^{5}$ Our objective in this paper is to show that in a setting with private information there is a second important aspect of delegation: the separation of control of economic decision-making from control of private information. The question is whether the delegation of bargaining to an agent can reduce the ability of the buyer to extract rents from the seller. We model this issue by assuming that the seller offers the agent a delegation contract that combines a public compensation component and a supplemental non-linear contract.

We provide several examples where bargaining is delegated by an informed principal to an agent and where non-linear contracts play a role in the relationship. The first example arises in the automobile industry, where manufacturers sell cars to dealerships rather than directly to consumers. The dealership buys each car at what is referred to as the invoice price and then bargains with customers over a final sale price. If a dealership becomes bankrupt, the manufacturer bears no liability beyond the warranty. In the United States, invoice prices are generally available to the buying public. However, the price paid by a customer can be below the invoice price because manufacturers offer dealerships holdbacks, which amount to a non-linear quantity bonus of $1 \%$ to $3 \%$ of the invoice price, and other monthly or yearly volume bonuses as part of the manufacturer-dealer contract. ${ }^{6}$ Arruñada etal (2001) documents discounts

\footnotetext{
${ }^{4}$ The information structure of our bargaining game is the same as in Ausubel and Deneckere
} (1989) except that in our model the seller is the informed. What is important for our results is the mismatch between exogenous bargaining power and the distribution of private information.

${ }^{5}$ This point has been made by Schelling (1956), Fershtman and Judd (1987), Katz (1991) and many others. More recently, Koçkesen and Ok (2004) apply forward induction arguments to complete information extensive form games and show that delegation by a weak party can yield strategic benefits.

${ }^{6}$ Consumer websites such as myAutoloan.com report that many manufacturer-to-dealer incentives 
from invoice prices to automobile dealers in Spain that range from 1\% to 3.5\% depending on the number of cars sold. Thus, automobile manufacturers, who have private information about production costs, delegate bargaining to their dealers via contracts whose compensation reflects both a publicly known component and a private non-linear component.

Similarly, WalMart delegates the purchase of over 1500 different categories of products to regional managers using a practice called category management. A manager is assigned to each product category in the store. This manager is responsible for negotiating with outside suppliers and is compensated on the basis of category profits. Part of the category's costs include shelf space costs as well as overhead charges that are paid to the retailer. ${ }^{7}$ Rather than giving the category manager discretion over how to use information such as the opportunity cost of shelf space, some retailers will "tie the hands" of a category manager by fixing its shelf space charge even though the manager's compensation includes other (possibly non-linear) components.

A third example concerns Lloyd's of London, which formed Equitas Ltd as a reinsurance company when faced with mounting liabilities from asbestos litigation in September $1996 .{ }^{8}$ Lloyd's capitalized Equitas with $£ 14.7$ billion in return for which Equitas reinsured all of the syndicate's pre-1993 asbestos liabilities. The contract between Lloyd's and Equitas further authorized Equitas to negotiate and settle any asbestos claims as Lloyd's agent (Hall, 2002). The motivation for this type of delegation was to address two problems: A number of court cases had weakened Lloyd's legal bargaining position in settling claims and the increased liability made it increasingly difficult to attract new capital to the syndicate. Unknown to policyholders was just how deep Lloyd's pockets were and this uncertainty was stalling settlement negotiations.

According to Standard and Poor's (2003), if Equitas were to become insolvent, Lloyd's would not be liable for any unmet liabilities. However, the contract creating Equitas also includes a formula for returning funds to Lloyd's after all claims have been settled and provisions for increasing Lloyd's payments to Equitas (American Names Association, 2000), something Lloyd's has done several times since Equitas's inception including a $£ 128$ million increase in 2006 (The Evening Standard (2006)). The

are not announced to the public and for the consumer it can be "impossible to know as much as the dealer knows about what the incentives are." (Horizon Digital Finance (2009))

${ }^{7}$ See Blattberg and Fox (1995-6) for details on category management practices.

${ }^{8}$ We thank Mort Kamien for bringing this example to our attention. 
initial capitalization of Equitas represented a public statement about its capacity to meet expected liabilities from the asbestos claims, about which Lloyd's has private information. However, the potential for additional capitalization, which is uncertain from the perspective of insureds, and the refund provisions of the contract are consistent with a non-linear component to the contract. One might question the credibility to insureds of the limited capitalization (especially given the increases Lloyd's has made) but, by 2004, Equitas had managed to settle two-thirds of initial claims at what has been referred to as "cheap deals with Equitas." (Fidler, 2004)

Finally, consider the problem of regulating a monopolist that is a subsidiary of a foreign-owned multinational (e.g., Gresik and Nelson (1994)). The subsidiary operates as a separate economic unit but is required to conduct business, such as trade in intermediate goods, with its parent on terms (i.e. transfer prices) usually set by the parent. In deciding how much to produce, the subsidiary is influenced by the taxes and regulations of its host government but also by the financial incentives provided by the parent. These financial incentives are determined by a combination of the transfer price set by the parent and by (non-linear) dividend and profit repatriation plans.

An intriguing feature of the principal-agent contract in all the examples is the combination of a public component and a less-public non-linear component. Why compensate car dealers or category managers with a combination of a piece rate (invoice price/space charges) and a nonlinear holdback or profit share? What is the significance of the initial capitalization of Equitas if Lloyd's is prepared to provide additional financing? After all, a well-designed non-linear contract can always incorporate the economic incentives of both the linear and non-linear compensation. ${ }^{9}$ In this paper, we will argue that this type of delegation contract, combining a public compensation component with a private non-linear contract, allows for competition via non-linear contracts between the informed party and the uninformed party that has very strong efficiency and surplus extraction properties. ${ }^{10}$

When an informed seller contracts with an agent to bargain on its behalf, the seller has essentially

${ }^{9}$ The transfer price example is more complex because, in addition to the piece rate/non-linear aspects of multinational finance, a transfer price shifts pre-tax dollars within the multinational while dividend payments shift after-tax dollars.

${ }^{10}$ Cai and Cont (2004) also analyze the seller's gains from delegating bargaining to an agent. In their model, it is the agent who has private information about his cost of effort and the amount of effort devoted to the bargaining process. They show that by imposing a minimum price on the agent, the seller can induce the agent to bargain more aggressively with the buyer. 
two options concerning the control of its private information. The first option involves the seller whispering a, possibly incorrect, private information report to the agent which specifies the agent's compensation. The agent is then responsible for bargaining with the customer. We refer to this option as "full delegation." Since the customer is still bargaining with a privately informed party, an inefficient quantity may still be traded. The commitment power of the seller's contract with the agent can constrain how successfully the customer can limit the agent's information rents and increases the set of equilibrium quantities. We show in section 4 that, with private information, multiple equilibria with distinct quantity schedules exist, including the first-best, making the equilibrium effect of commitment indeterminate under full delegation. To argue that full delegation is beneficial for the firm relative to direct bargaining one could invoke some sort of equilibrium selection criteria as in Koçkesen and Ok (2004). Rather than following this possibility, we consider a second option.

With this second option, the seller offers the agent a set of non-linear contracts. The specific contract the seller uses to compensate the agent is based on a public, payoff-relevant type announcement made before the agent and the customer bargain. The public nature of the type report implies that the bargaining game between the agent and the customer is now one of complete but imperfect information because the customer can now condition its offer on the public report. Because the seller's type does not enter the buyer's or the agent's payoff and the seller does not make any other choices following the public report, the seller's incentive is to maximize the total surplus from agent-buyer bargaining. As a result, the buyer and the agent will trade a first-best quantity for all seller types. Thus in equilibrium, partial delegation guarantees that the seller can strictly implement the first-best quantity. Furthermore, the seller collects all the surplus up to a constant with the remainder accruing to the customer. This gives all but the worst type of the informed seller a strict incentive to delegate in this manner. Since the agent in our model does not possess better information than the seller and since we do not allow the act of delegation to weaken the customer's bargaining power, this result is due to the strategic value of partial delegation and not because of any contractual or informational incompleteness experienced by the informed party nor because of any strategic signalling associated with the act of delegation.

The nature of partial delegation equilibria raises two important questions. Can an informed party credibly commit to only use a public channel to communicate type information to an agent? What is the role of private information? The first question focuses on the critical difference between our full delegation and partial delegation settings. Partial delegation requires that the informed seller be able to commit to communicating private information to its agent only through a public channel. If private communication is possible, even with public communication, then we are back in a full delegation 
environment. We argue that because of the strong implementation feature of our partial delegation game, the informed seller has no strict incentive to engage in private communication. Any incentive the seller would want to create for the agent through private communication can also be created through the nonlinear component of the agent's contract. By avoiding private type communication, the seller eliminates any equilibrium coordination issues present under full delegation.

The second question focuses on the role of private information. On the surface it might appear that our main result does not rely on private information and is essentially a restatement of the ideas of Fershtman and Judd (1987). In fact, private information is essential for our results. Without private information, there is no information control issue. It is true that, in the complete information version of our bargaining game with delegation, among all differentiable equilibria, the quantity is unique and firstbest. But this is also true for the complete information version of our direct bargaining game (i.e., between the firm and the customer). Our results emphasize the importance of separating the agent's control of economic decision-making from the agent's control of private information as a response to the distortions private information creates. The extant wisdom about the impact of private information is that it introduces distortions in response to information rent effects. Our analysis shows that, under partial delegation, an informed party will earn higher information rents than it could under a direct bargaining scenario in which it has a weak bargaining position, and that it can do so without reliance on the very subtle strategic communications needed to resolve equilibrium coordination issues.

In the next section, we set up a simple model of principal to principal (seller to customer) bargaining to establish a baseline. In Section 3, we then permit the informed seller to partially delegate bargaining responsibilities to an agent and characterize the equilibria of the bargaining game. In Section 4, we analyze a game with fully delegated bargaining. We offer concluding comments in Section 5.

\section{A Model of Bargaining Between Two Principals.}

We begin by describing the strategic interaction between an informed seller and an uninformed buyer negotiating the terms of trade for $q$ units of a product in the context of a well-known model. The buyer's gross benefit from trade is $b(q)$ and the seller has production costs $c(q, \theta)$, where $\theta$ is the seller's private information about cost. Throughout the paper, it will be common knowledge that $\theta$ is drawn from

the distribution $F(\cdot)$ with compact support $[\underline{\theta}, \bar{\theta}]$ and strictly positive density, $f(\cdot)$. The total surplus from any transaction is $w(q, \theta) \equiv b(q)-c(q, \theta)$.

We adopt the following (standard) assumptions regarding the payoff and distribution functions:

Assumption 1. a. $c(q, \theta)$ is convex and increasing in $q$ and $c(0, \theta)=0$.

b. $b(q)$ is strictly concave and strictly increasing in $q, b(0)=0$, and $b^{\prime}(0)-c_{q}(0, \theta)>0$. 


\section{c. $c(; \theta)$ and $c_{q}(; \theta)$ are strictly increasing in $\theta$. \\ d. $F(\theta) / f(\theta)$ is strictly increasing in $\theta$. \\ e. $c_{\theta q}(q, \theta)$ is non-increasing in $q$ and $\theta$.}

Assumptions (a) - (c) ensure that there will be a unique first best quantity, $q^{F}(\theta)>0$, that solves

$w_{q}(q, \theta)=0$. Assumption (c) guarantees that $q^{F}(\theta)$ and $w(q, \theta)$ are strictly decreasing in $\theta$. Assumption (d) implies that our model satisfies the usual single crossing property. Assumption (e) provides sufficient conditions on seller costs for incentive efficient quantities to be non-increasing in $\theta$. Most models simply assume $c$ is linear in $\theta$ and $q$.

For any Bayes-Nash equilibrium of any bargaining game, the resulting allocation can be described by a pair $(q(\theta), t(\theta))$ where $q(\theta)$ denotes the quantity traded and $t(\theta)$ is the amount the buyer pays the seller when the seller's type is $\theta$. In characterizing the equilibrium of a bargaining game we invoke Rochet's (1985) Taxation Principle, which allows us to represent any equilibrium allocation by a non-linear price contract $T(q)$ that represents the payment the buyer makes to the seller in order to buy $q$ units. The implied bargaining game is one in which the parties negotiate over $T(\cdot)$ and then the seller chooses $q$. For any allocation $(q(\theta), t(\theta))$ there exists a non-linear contract such that in equilibrium the seller chooses $q(\theta)$ and $T(q(\theta))=t(\theta)$. Thus, deriving the set of non-linear contracts that maximize an interim weighted social welfare function subject to incentive constraints to describe incentive efficient bargaining is equivalent to the standard direct revelation approach. We present the non-linear contract approach for consistency with the analysis in sections 3 and 4 where it will be necessary to focus on nonlinear contracts.

For any contract $T(q)$, total profit for the buyer and for the seller is

$$
B(q)=b(q)-T(q) \text { and } s(q, \theta)=T(q)-c(q, \theta) .
$$

Assuming that $T(q)$ is differentiable, any strictly positive profit-maximizing quantity for the seller, $q(\theta)$, will satisfy

$$
T^{\prime}(q(\theta))=c_{q}(q(\theta), \theta) \text { and } T^{\prime \prime}(q(\theta)) \leq c_{q q}(q(\theta), \theta) .
$$

Denote the seller profit generated at its optimal quantity choice by $S(\theta) \equiv s(q(\theta), \theta)$. Differentiation of $S(\theta)$ yields the incentive compatibility conditions that $q(\theta)$ must satisfy:

$$
d S(\theta) / d \theta=-c_{\theta}(q(\theta), \theta)=w_{\theta}(q(\theta), \theta)<0
$$

and

$$
\mathrm{q}^{\prime}(\theta) \leq 0 .
$$

By integrating (3), seller profit must equal 


$$
S(\theta)=S(\bar{\theta})-\int_{\theta}^{\bar{\theta}} w_{\theta}(q(t), t) d t
$$

In order for the buyer and the seller to be willing to participate in a bargaining game, it must yield a non-negative payoff to each party. For the seller, it follows from (5) that participation is guaranteed for all values of $\theta$ as long as $S(\bar{\theta}) \geq 0$. Similarly, participation by the buyer yields a non-negative expected return if

$$
\mathscr{E}_{\theta}[w(q(\theta), \theta)-S(\theta)] \geq 0
$$

Incentive efficiency, as defined by Holmström and Myerson (1983), describes the set of quantitymoney allocations $(q(\theta), T(q(\theta))$ for which there exists no other incentive compatible allocations $\left(q^{1}(\theta), T^{1}\left(q^{1}(\theta)\right)\right.$ that yields higher expected profit for the buyer without giving any seller type lower profit or higher profit for any seller type without giving the buyer lower expected profit. Myerson (1985) and Wilson (1985) show that this incentive constrained Pareto frontier can be described by maximizing the weighted welfare function

$$
\hat{W}=\int_{\underline{\theta}}^{\bar{\theta}}[\alpha(\theta) S(\theta)+\gamma B(q(\theta))] f(\theta) d \theta
$$

subject to incentive compatibility constraints, (3) and (4), and participation constraints, (6) and $S(\bar{\theta}) \geq 0$. The non-negative weights, $\alpha(\cdot)$ and $\gamma$, are normalized so that

$$
\gamma+\int_{\underline{\theta}}^{\bar{\theta}} \alpha(\theta) f(\theta) d \theta=1
$$

Intuitively, these welfare weights reflect the relative bargaining power of the buyer and seller. We provide a complete characterization of all incentive efficient allocations with strictly decreasing quantity schedules in the Appendix as the general solution relates to some of our analysis in section 4. However, to establish the main point of this paper, it is sufficient to focus on two specific welfare weight distributions and hence on two specific constrained Pareto efficient allocations: (i) $\gamma=1$ and $\alpha(\cdot) \equiv 0$ and (ii) $\gamma=0$ and $\alpha(\cdot) \equiv 1$. We describe the associated allocations in the following proposition.

Proposition 1. If $\gamma=1$ and $\alpha(\cdot)=0$, the incentive efficient allocation implies $S(\bar{\theta})=0$ and $w_{q}(q(\theta), \theta)=(F(\theta) / f(\theta)) w_{\theta q}(q(\theta), \theta)$. If $\gamma=0$ and $\alpha(\cdot) \equiv 1$, the incentive efficient allocation implies $S(\bar{\theta})=w\left(q^{F}(\theta), \theta\right)$ and $q(\theta)=q^{F}(\theta)$.

The first result in Proposition 1 is the solution to the standard principal-agent type problem in which the informed party with the highest possible type earns zero profit and all other types earn positive 
profit. Only the lowest type trades a first-best quantity. All other types trade an inefficiently low quantity. This allocation is the unique equilibrium allocation that would arise in a game in which the buyer has the power to credibly make a take-it-or-leave-it offer to the seller. For future reference, we denote this quantity schedule by $q^{0}(\theta)$. The properties of this allocation are robust in the sense that $S(\bar{\theta})=0$ and $q(\theta)<q^{F}(\theta)$ for all $\theta>\underline{\theta}$ as long as the average seller weight is less than the buyer weight, i.e., $\gamma>1 / 2$, and low seller type profit is weighted more than high seller type profit, i.e., $\alpha^{\prime}(\cdot)<0$. The important feature driving this result is the fact that the privately informed party is in the weaker bargaining position due to exogenous factors. The seller's bargaining power is weakest when $\gamma=1$.

The second result in Proposition 1 is included to remind the reader that first-best quantities are incentive efficient for some welfare weights. The extreme weights in Proposition 1 correspond to a bargaining game in which the informed seller has the ability to make a take-it-or-leave-it offer to the buyer. Since the seller can always extract all of the surplus generated by trade, it is incentive compatible for the seller to offer a contract that induces first-best quantities. ${ }^{11}$ This result is also robust to variations in the welfare weights such that $\alpha(\theta)$ is a constant greater than $1 / 2$ (and $\gamma<1 / 2$.) For $\alpha(\theta)<1$, $S(\bar{\theta})$ is less than or equal to $w\left(q^{F}(\bar{\theta}), \bar{\theta}\right)$ as the seller now earns all of the surplus minus the buyer's constant share. This result is indicative of a bargaining outcome when the ownership of private information and exogenous bargaining power are aligned.

\section{Partial Delegation.}

We now allow the seller to hire an agent who is given the authority to negotiate a price and quantity. In making the decision to delegate negotiating authority to the agent, the seller must decide how to share its private information with its agent. In this section, we consider a partial delegation scenario in which the seller makes a public type report to both the agent and the buyer. We use the term "partial delegation" because the seller delegates the responsibility to the agent for deciding how much to sell to the buyer but retains control of how information is released to the buyer. In the next section we consider the "full delegation" case in which the seller communicates type information to the agent privately. ${ }^{12}$

\footnotetext{
${ }^{11}$ See Spulber (1988) and Ausubel and Deneckere (1989) for more details.

${ }^{12}$ Previous efforts to model delegation with a privately informed principal include Katz (1991)
} and Caillaud and Hermalin (1993). Katz offers an example that suggests private information can provide a motive for delegation. In his paper, the agent is uninformed when offered a contract by the informed principal but the true value of this private information is truthfully revealed to the agent and the outside principal prior to any output decisions. In contrast, the seller in our model retains the ability to distort its 
We study this idea of partial delegation by deriving the Perfect Bayes Nash equilibria of the following two-stage game. In the first stage, the seller hires an agent. The agent receives a contract of the form $T^{s}(q, \kappa)$ where $\kappa$ denotes a public type report, also made in stage one. $T^{s}(q, \kappa)$ represents a payment from the agent to the seller. For example, $\kappa$ could be the invoice price of a car and any non-linear component to $T^{s}$ could correspond to a holdback. The buyer cannot observe the contract the seller offers the agent. In the second-stage, the agent and the buyer negotiate by having the buyer make the agent a take-it-or-leave-it offer which the agent either accepts or rejects. This last feature maintains the distribution of bargaining power from Proposition 1 that resulted in a second-best level of trade. Because $\kappa$ is a public report, the buyer can condition its offer on $\kappa$ which means we can represent the equilibrium in this second stage by a collection of non-linear contracts (indexed by $\kappa), T^{b}(q, \kappa)$, and an optimal quantity choice by the agent.

Given the non-linear contract approach to formalizing this game, a strategy for the seller consists of the contract each type of seller offers the agent, $T^{s}(q, \kappa ; \theta)$, and the public report each type makes, $\kappa(\theta)$. A strategy for the buyer is a report-contingent contract $T^{b}(q, \kappa)$. A strategy for the agent is a quantity choice given the specific contracts, $T^{s}(q, \kappa ; \theta)$ and $T^{b}(q, \kappa)$, which we denote using short-hand notation by $q^{a}(\kappa ; \theta)$ where the reliance on $\theta$ reflects the fact that different seller types might offer different contracts and hence induce different quantities even though the agent does not know $\theta$.

Given a pair of contracts, $T^{s}$ and $T^{b}$, a report, $\kappa$, and a quantity, $q$, the seller's profit equals

$$
s(q, \kappa, \theta)=T^{s}(q, \kappa ; \theta)-c(q, \theta),
$$

the buyer's profit equals

information report to both the agent and the buyer. Caillaud and Hermalin examine the benefits of employing an agent in a signalling model. In their paper, as soon as an agent is hired, the informed principal becomes uninformed and the agent now controls the private information. This creates countervailing incentives due to a tension between the screening motives of the delegating principal to learn the agent's type and the principal's signalling motives. Caillaud and Hermalin show that these countervailing incentives can result in a higher equilibrium payoff to the informed principal. Again, in contrast, the informed seller in our model never becomes uninformed. Alonso and Matouschek (2007) focus on the optimality of contracts in which the principal retains the right to overrule the agent on certain decisions. They show that full delegation is never optimal but, similar to the above papers, the principal in their model is uninformed. 


$$
B(q, \kappa)=b(q)-T^{b}(q, \kappa),
$$

and the agent's profit is

$$
a(q, \kappa ; \theta)=T^{b}(q, \kappa)-T^{s}(q, \kappa ; \theta) .
$$

Note that agent profit depends on the seller's true type only through the seller's choice of contract.

We require the buyer and the seller contracts to be differentiable. This turns out to be a critical assumption. When both principals are fully informed, this differentiability condition implies that the equilibrium quantity traded equals $q^{F}(\theta)$ in any Nash equilibrium in which a positive quantity is traded for all $\theta$. If we admit non-differentiable schedules, a continuum of equilibrium quantities will exist even under complete information. Thus, it would not be surprising to show that adding private information to a game with non-differentiable equilibria also resulted in a large set of equilibria. Instead we wish to focus on an environment in which the complete information equilibrium quantity profile is unique.

\subsection{Equilibrium Definitions}

Because the public report creates signalling incentives for the seller, we will focus on the Perfect Bayes Nash equilibria of this game. Denote the equilibrium quantity traded by $q^{*}(\theta)$ and the equilibrium payments by $T^{b}\left(q^{*}(\theta), \kappa(\theta)\right)$ and $T^{s}\left(q^{*}(\theta), \kappa(\theta) ; \theta\right)$.

Given any pair of contracts and any public report, the agent's optimal quantity choice will maximize (11) subject to $a(q, \kappa ; \theta) \geq 0$ and will satisfy

$$
T_{q}^{b}\left(q^{a}(\kappa ; \theta), \kappa\right)=T_{q}^{s}\left(q^{a}(\kappa ; \theta), \kappa ; \theta\right) \text { and } T_{q q}^{b}\left(q^{a}(\kappa ; \theta), \kappa\right) \leq T_{q q}^{s}\left(q^{a}(\kappa ; \theta), \kappa ; \theta\right)
$$

as long as $a\left(q^{a}(\kappa ; \theta), \kappa ; \theta\right) \geq 0$. In equilibrium, $q^{a}(\kappa(\theta) ; \theta)$ must equal $q^{*}(\theta)$.

Since $\theta$ affects the agent's profit only through the contract offered by the seller, we do not need to specify beliefs for the agent about $\theta$. We do however need to specify the buyer's beliefs which we denote by $\zeta(\theta, \kappa)$ where $\zeta$ is a distribution function. These beliefs need to reflect the fact that the seller can pool in two ways: different seller types, $\theta$ and $\theta^{\prime}$, could offer the same contracts so that $T^{s}(q, \kappa ; \theta)=T^{s}\left(q, \kappa ; \theta^{\prime}\right)$ or they could make the same type report so that $\kappa(\theta)=\kappa(\theta)$. If seller types $\theta$ and $\theta^{\prime}$ make different public reports, that is $\kappa(\theta) \neq \kappa\left(\theta^{\prime}\right)$, the buyer's beliefs will reflect the seller's contract with certainty. The same is true if different types offer the same report and the same contract. However, if different seller types offer different contracts but issue the same public report, then the buyer's beliefs must reflect uncertainty over the contract the seller gives the agent. To describe this uncertainty, suppose there exists a set of types $P(\bar{\kappa})$ such that for all $\theta \in P(\bar{\kappa}), \kappa(\theta)=\bar{\kappa}$. Using Bayes Rule, the buyer's beliefs about the seller's type can be denoted by $\zeta(\theta, \bar{\kappa})=F(\theta \mid P(\bar{\kappa}))$. For each value of $\kappa$ that no seller type uses in equilibrium, any beliefs are feasible. Then the buyer's problem is, for each seller strategy ( $\left(T^{s}(q, \kappa ; \theta), \kappa(\theta)\right)$, to choose $T^{b}(q, \kappa)$ to maximize 


$$
\int_{\theta \in P(\kappa)}\left[b\left(q^{a}(\kappa ; \theta)\right)-T^{b}\left(q^{a}(\kappa ; \theta), \kappa\right)\right] d \zeta(\theta, \kappa)
$$

subject to (i) For each $\theta \in P(\kappa)$, if $q^{a}(\kappa ; \theta)>0$, then

$$
q^{a}(\kappa ; \theta) \in \operatorname{argmax}_{q} T^{b}(q, \kappa)-T^{s}(q, \kappa ; \theta) \text { s.t. } T^{b}(q, \kappa) \geq T^{s}(q, \kappa ; \theta) \text {, and }
$$

(ii) For each $\theta \in P(\kappa)$, if $q^{a}(\kappa ; \theta)=0$, then $T^{b}(q, \kappa)<T^{s}(q, \kappa ; \theta)$ for all $q>0$.

Although the buyer chooses her contract after the seller, the fact that the buyer cannot observe the seller's contract means the seller cannot influence the buyer's selection of a contract in a Stackelberg fashion. This means the seller's problem is for each $T^{b}(q, \kappa)$ to choose $\left(T^{s}(q, \kappa ; \theta), \kappa(\theta)\right)$ to maximize

$$
T^{s}\left(q^{a}(\kappa ; \theta), \kappa ; \theta\right)-c\left(q^{a}(\kappa ; \theta), \theta\right)
$$

subject to (i) if $q^{a}(\kappa ; \theta)>0$, then

$$
q^{a}(\kappa ; \theta) \in \operatorname{argmax}_{q} T^{b}(q, \kappa)-T^{s}(q, \kappa ; \theta) \text { s.t. } T^{b}(q, \kappa) \geq T^{s}(q, \kappa ; \theta) \text {, and }
$$

(ii) if $q^{a}(\kappa ; \theta)=0$, then $T^{b}(q, \kappa)<T^{s}(q, \kappa ; \theta)$ for all $q>0$.

Thus, a (differentiable) Perfect Bayes Nash Equilibrium (PBNE) is a strategy profile $\left(\left(T^{s}, \kappa\right), T^{b}, q^{a}\right)$ and beliefs $\zeta(\theta, \kappa)$ such that $\left(T^{s}, \kappa\right)$ solves (SP), $T^{b}$ solves (BP) given $\zeta(\theta, \kappa), q^{a}$ maximizes (11) subject to non-negative agent profit, and $\zeta(\theta, \kappa)$ is defined as above.

With unobservable contracts, there always exist no-trade equilibria in which the seller's (buyer's) contract guarantees the buyer (seller) will earn negative profit no matter what quantity the agent chooses. This can be true for all $\theta$ or for some $\theta$. For such $\theta$, the buyer and the seller each have the incentive to make sure the agent does not trade. Consistent with the rest of the common agency literature, we focus only on positive trade equilibria for all $\theta$, that is, $q^{*}(\theta)>0$ for all $\theta$. We will refer to these equilibria as "positive-trade, differentiable, partial delegation" equilibria.

The case in which the seller can offer a contract $T^{s}$ to the agent that the buyer can observe serves as a useful benchmark. By committing to an observable contract, the seller is able to force the agent and the buyer into a specific quantity schedule by making all other quantities unprofitable for the agent. Thus, all differentiable Bayes Nash equilibria result in $q^{F}(\theta)$ being traded for all $\theta$, zero agent profit, and zero buyer profit. The first-best quantity remains the unique equilibrium quantity even if the buyer does not have sufficient bargaining power to make take-it-or-leave-it offers. If $\gamma>1 / 2$, any incentive efficient bargaining mechanism would result in the buyer earning all of the surplus, which the seller can extract with partial delegation. If $\gamma=1 / 2$ so that any division of the surplus between the buyer and the agent is efficient, the seller's best response to any positive-quantity bargaining outcome is still to maximize the surplus by inducing $q^{F}(\theta)$ and to extract all the surplus via the agent. Restricting attention to the case in which the buyer has all the bargaining power serves only to simplify the analysis. 


\subsection{Equilibrium characterization.}

The examples in the introduction and in Katz (1991) are all consistent with seller contracts the buyer cannot observe. Assuming the seller's contract is unobservable to the buyer is formally equivalent to assuming the buyer and seller simultaneously announce non-linear contracts to the agent. Unlike in section 2 where we had the option of restricting attention to direct mechanisms or to non-linear contracts, it is now imperative that we focus on competition in non-linear contracts. Simultaneous contracting implies we are studying a class of common agency games. Martimort and Stole (2002) and Peters (2002, 2003) show that restricting attention to competition in non-linear contracts involves no loss of generality because of the quasi-linear preferences of the principals and the agents. The same cannot be said about competition in direct mechanisms. To ensure that our results are not driven by ad hoc contract space restrictions, it is now important that we allow the buyer and the seller to compete in non-linear contracts.

First, consider the seller's best response to any buyer strategy, $T^{b}(q, \kappa)$. In order to focus on positive trade equilibria, define a buyer contract $T^{b}(q, \kappa)$ to be feasible if for each $\theta$ there exists $\kappa \in[\underline{\theta}, \bar{\theta}]$ and $q>0$ such that $b(q) \geq T^{b}(q, \kappa) \geq c(q, \theta)$. Feasibility implies that each seller type will want to induce the agent to trade a strictly positive quantity for some $\kappa$. We include the upper bound to rule out buyer contracts dominated for the buyer by those that result in no trade and for symmetry with an analogous condition on seller contracts introduced below. Denote the seller's optimal quantity schedule by $q^{s}(\theta)$ and the seller's optimal public report schedule by $\kappa^{s}(\theta)$. Agent profit must be zero under any seller best response that implies $q^{s}>0$. Thus, in response to a feasible buyer contract, the seller will extract any agent surplus by setting $T^{s}\left(q^{s}(\theta), \kappa^{s}(\theta) ; \theta\right)=T^{b}\left(q^{s}(\theta), \kappa^{s}(\theta)\right)$ for all $\theta$ and will ensure that the agent has the incentive to accept $q^{s}(\theta)$ by making sure $T^{s}\left(q, \kappa^{s}(\theta) ; \theta\right) \geq T^{b}\left(q, \kappa^{s}(\theta)\right)$ for all $q$ and for all $\theta$. Zero agent profit implies the seller's payoff can be written as

$$
s\left(q^{s}, \kappa^{s}, \theta\right)=T^{b}\left(q^{s}, \kappa^{s}\right)-c\left(q^{s}, \theta\right) .
$$

Eq. (13) is maximized when the buyer's marginal payment equals the seller's marginal cost.

Lemma 1: If $T^{b}(q, \kappa)$ is a feasible contract, then the seller's best response (if it exists) must satisfy

$$
\begin{aligned}
& T^{s}\left(q^{s}(\theta), \kappa^{s}(\theta) ; \theta\right)=T^{b}\left(q^{s}(\theta), \kappa^{s}(\theta)\right) \text { for all } \theta, \\
& T_{q}^{b}\left(q^{s}(\theta), \kappa^{s}(\theta)\right)=c_{q}\left(q^{s}(\theta), \theta\right) \text { for all } \theta \text {, and } \\
& T^{s}\left(q, \kappa^{s}(\theta) ; \theta\right) \geq T^{b}\left(q, \kappa^{s}(\theta)\right) \text { for all } q \text { and for all } \theta .
\end{aligned}
$$

Note that Lemma 1 does not include any conditions on $\kappa^{s}(\theta)$. We will address such optimality conditions later in our analysis.

Second, consider the buyer's best response to any seller strategy $\left(T^{s}(q, \kappa ; \theta), \kappa(\theta)\right)$. Similar to the analysis of the seller's problem, define a seller contract $T^{s}(q, \kappa ; \theta)$ to be feasible if for each $\theta$ there exists 
$q>0$ such that $b(q) \geq T^{s}(q, \kappa(\theta) ; \theta) \geq c(q, \theta)$. Denote the buyer's optimal quantity schedule given $\kappa$ by $q^{b}(\theta)$.

Two cases need to be considered with respect to $\kappa(\theta)$ : full separation and partial or full pooling. With full separation, there exist no types $\theta^{\prime}$ and $\theta^{\prime \prime}$ such that $\kappa(\theta)=\kappa\left(\theta^{\prime}\right)$. In this case, $\zeta(\theta \mid \kappa(\theta))=1$ if $\theta=\theta$ and zero if $\theta^{\prime} \neq \theta$. If $\kappa \neq \kappa(\theta)$ for all $\theta$, then buyer beliefs are unrestricted. If the seller types fully separate with the public report, there is no added advantage or disadvantage to separating over contracts.

Lemma 2. If the seller uses a fully separating public report schedule and $T^{s}(q, \kappa ; \theta)$ is a feasible contract, then the buyer's best response (if it exists) must satisfy

$$
\begin{aligned}
& T^{s}\left(q^{b}(\theta), \kappa(\theta) ; \theta\right)=T^{b}\left(q^{b}(\theta), \kappa(\theta)\right) \text { for all } \theta, \\
& T_{q}^{s}\left(q^{b}(\theta), \kappa(\theta) ; \theta\right)=b^{\prime}\left(q^{b}(\theta), \kappa(\theta)\right) \text { for all } \theta, \\
& T^{s}(q, \kappa(\theta) ; \theta) \geq T^{b}(q, \kappa(\theta)) \text { for all } q \text { and for all } \theta \text {. }
\end{aligned}
$$

Lemma 2 implies that a fully separating reporting strategy by the seller gives the buyer the incentive to maximize the residual surplus, $T^{s}(q, \kappa(\theta) ; \theta)-b(q)$. This incentive arises because report separation supports updated buyer beliefs that allow the buyer to leave the agent with zero profit for each $\theta$. Thus, the buyer's best response when the seller's public report is fully separating will result in zero agent profit, the seller's marginal payment from the agent equal to the buyer's marginal benefit, and non-positive agent profit for all $q$.

With partial or full pooling over $\kappa$, there exist types $\theta$ and $\theta^{\prime}$ such that $\kappa(\theta)=\kappa(\theta)$. If these types choose identical contracts, Lemma 2 still applies. Thus, if a positive-trade equilibrium involves full separation with $\kappa$ or pooling on contracts for all seller types that pool on the same $\kappa$, then $q^{*}(\theta)$ must equal $q^{F}(\theta)$ for all $\theta$ as only $q^{F}(\theta)$ satisfies (12), (15), and (18).

The remaining possibility is that seller types that pool on $\kappa$ do not offer the same contract. Lemma 3 which is formally stated and proved in the appendix implies that if in equilibrium some seller types pool on their reports but separate on their contracts, the quantity the buyer procures from those types via the agent must satisfy (18). The intuition for Lemma 3 is based on three observations. First, with this type of pooling, the buyer will not be able to determine in equilibrium which contract the agent faces and as a result may have to pay the agent an information rent. Let $P(\bar{\kappa})$ denote the set of seller types who announce $\bar{\kappa}$ in equilibrium and suppose that some of these types offer the agent different contracts. In order for the buyer to induce the agent to sell $q^{b}(\theta)$ units for all $\theta \in P(\bar{\kappa})$, it must be that $T^{b}\left(q^{b}(\theta), \bar{\kappa}\right)-T^{s}\left(q^{b}(\theta), \bar{\kappa} ; \theta\right)=\max _{\mathrm{q}}\left[T^{b}(q, \bar{\kappa})-T^{s}(q, \bar{\kappa} ; \theta)\right]$. The agent will earn an information rent due to the seller pooling on $\kappa$ but separating on $T^{s}$ if, for some $\theta \in P(\bar{\kappa})$, the buyer chooses a contract that implies $\max _{\mathrm{q}}\left[T^{b}(q, \bar{\kappa})-T^{s}(q, \bar{\kappa} ; \theta)\right]>0$. Second, in any positive trade, differentiable PBNE we know 
from Lemma 1 that the agent must earn zero profit. But this means the buyer's contract, conditional on hearing $\bar{\kappa}$, can never exceed, for any $q$, the amount the agent must pay any of the seller types who announce $\bar{\kappa}$. Therefore, for all $q$ and for all $\theta \in P(\bar{\kappa}), T^{b}(q, \bar{\kappa}) \leq \min T^{s}(q, \bar{\kappa} ; \theta)$ and for all $\theta \in P(\bar{\kappa})$, $T^{b}\left(q^{b}(\theta), \bar{\kappa}\right)=T^{s}\left(q^{b}(\theta), \bar{\kappa} ; \theta\right)$. Third, given positive-trade, differentiable equilibrium contracts that we know from the second observation must imply zero agent profit at $q^{b}(\theta)$ for all $\theta \in P(\bar{\kappa})$, now assume that, for some $\hat{\theta} \in P(\bar{\kappa})$, (18) does not hold, that is, $b^{\prime}\left(q^{b}(\hat{\theta})\right) \neq T_{q}^{s}\left(q^{b}(\hat{\theta}), \bar{\kappa} ; \hat{\theta}\right)$. The proof shows that this assumption contradicts the idea that the buyer maximizes its expected profit by $q^{b}(\theta)$ given $\bar{\kappa}$. Namely, $b^{\prime}\left(q^{b}(\hat{\theta})\right) \neq T_{q}^{s}\left(q^{b}(\hat{\theta}), \bar{\kappa} ; \hat{\theta}\right)$ means that a small change away from $q^{b}(\hat{\theta})$ will have a first-order increase in the buyer's profit when the seller's type is $\hat{\theta}$. To realize this increased profit, the buyer will have to pay the agent a positive rent to give the agent an incentive to select this deviation quantity. However, this rent may be earned by the agent for $\theta \in P(\bar{\kappa})$ not equal to $\hat{\theta}$ as well. Differentiability of the seller contracts implies the marginal rent associated with inducing such a deviation quantity is zero at $q^{b}(\hat{\theta})$. Since the rent increase associated with a small deviation from $q^{b}(\hat{\theta})$ is second-order in magnitude, the deviation quantity will increase the buyer's expected profit when $\bar{\kappa}$ is announced. Thus, $q^{b}(\hat{\theta})$ could not have been part of the buyer's best response. Lemmas 1 and 3 together imply that if a positive trade, differentiable partial delegation equilibrium involves public report pooling and contract separation for some seller types that $b^{\prime}\left(q^{*}(\theta)\right)=c_{q}\left(q^{*}(\theta), \theta\right)$. Thus, the only possible strictly positive equilibrium quantity is $q^{F}(\theta)$.

To complete our derivation of equilibria, we need to identify non-linear contracts that constitute an equilibrium as so far we have only discussed necessary conditions. There exist many contracts that will work. We describe one such pair in the appendix. Equilibrium payoffs for the buyer and the seller are also not unique. If one defines the seller's equilibrium profit as $\Sigma(\theta)=s\left(q^{a}(\kappa(\theta)), \kappa(\theta), \theta\right)$, then by the Envelope Theorem, $\Sigma^{\prime}(\theta)=s_{\theta}\left(q^{F}(\theta), \kappa(\theta), \theta\right)=w_{\theta}\left(q^{F}(\theta), \theta\right)$ and $d w\left(q^{F}(\theta), \theta\right) / d \theta=w_{\theta}\left(q^{F}(\theta), \theta\right)$. Thus, the buyer's equilibrium profit, $w\left(q^{F}(\theta), \theta\right)-\Sigma(\theta)$, must equal a constant but it can take on values between 0 and $b\left(q^{F}(\bar{\theta})\right)-c\left(q^{F}(\bar{\theta}), \bar{\theta}\right)$. In the appendix, constant buyer profit is achieved by the buyer paying $b(q)$ minus a constant $\phi$. The seller contract charges the agent $b(q)$ - $\phi$ plus a non-negative penalty term. The non-negative penalty term is zero at $q^{F}(\kappa)$ and is structured so that the agent earns strictly negative profit by selling any quantity other than $q^{F}(\kappa)$ and so that seller profit is maximized by reporting $\kappa=\theta$. Finally, we also need to rule out the possibility of mixed strategy equilibria in which either the buyer and/or the seller randomize in a way that results in a distribution of equilibrium quantities for some measurable subset of types. ${ }^{13}$ Proposition 2 summarizes the above analysis.

\footnotetext{
${ }^{13}$ Suppose the buyer were to randomize over the contracts $T_{1}^{b}$ and $T_{2}^{b}$. Agent incentive
} 
Proposition 2. If the buyer can observe the seller's type report but not the contract the seller offers the agent, then all positive trade, differentiable partial delegation equilibria result in first-best trade, $q^{F}(\theta)$. The seller earns profit $\Sigma(\theta)=w\left(q^{F}(\theta), \theta\right)$ - K and the buyer earns profit of $K$, where $K \in\left[0, w\left(q^{F}(\bar{\theta}), \bar{\theta}\right)\right]$.

Proposition 2 describes all possible positive trade, differentiable equilibrium outcomes. Two sources of multiplicity exist. The first is due to the fact that any strictly increasing reporting strategy is strategically equivalent to $\kappa(\theta)=\theta$ and the second is due to the fact that there is a range of positive surplus levels the buyer can earn. It should be noted that even though the seller's contract is unobservable to the buyer, the seller does not have an incentive to renegotiate the contract with the agent once the buyer's offer has been observed. The seller obtains the maximum surplus available given the buyer's offer, and thus a mutually beneficial renegotiation between buyer and seller is not possible.

With observable contracts, the seller clearly benefits from committing to a partial delegation contract because all the surplus is extracted from the buyer. It turns out that there is a similar benefit from partial delegation with unobservable contracts, even though the buyer can retain some surplus, as long as the buyer has more of the bargaining power than the seller or the agent. The difference in the seller's equilibrium profit with and without partial delegation when the buyer has the majority of bargaining power is $\Sigma(\theta)-S(\theta)$ or

$$
w\left(q^{F}(\bar{\theta}), \bar{\theta}\right)-K+\int_{t=\theta}^{\bar{\theta}}\left[w_{\theta}\left(q^{0}(t), t\right)-w_{\theta}\left(q^{F}(t), t\right)\right] d t .
$$

By Proposition 2, the difference of the first two terms in (20) is non-negative and for $\theta<\bar{\theta}$, the integral is strictly positive. Thus, (20) is strictly positive for all $\theta<\bar{\theta}$.

Proposition 3. Suppose the buyer has more bargaining power than the seller or the agent. For all seller

compatibility may not allow the seller to drive the agent to zero rent and implement the seller's first-best quantities under each buyer contract. If a differentiable mixed strategy equilibrium were to exist in which some of the possible combinations of contracts the agent faces induce different quantities, some of the equilibrium quantities would be first-best from the seller's perspective and some would be second-best. Similarly, some of the quantities would be first-best from the buyer's perspective and some would not. It turns out that the buyer and the seller are never able to "agree" as to which contract pairs should yield first-best quantities and which should not (from the perspective of each individual trader). Details of this analysis are available from the authors on request. 
types less than $\bar{\theta}$, all positive-trade, differentiable equilibria of the partial delegation game result in higher equilibrium seller profit than direct bargaining even if the seller's contract to the agent is not observable.

It is important to recognize that partial delegation does not expand the incentive constrained Pareto frontier. It does not constitute a Pareto improvement over direct seller-to-buyer bargaining. Rather, because Proposition 1 (following on Spulber (1998)) establishes welfare weights for which firstbest quantities are incentive efficient, the correct interpretation of Propositions 2 and 3 is one of implementation. We elaborate on this idea in Section 4. When direct bargaining puts an informed trader in a weak bargaining position, partial delegation allows the informed trader to implement a preferred incentive efficient allocation.

\subsection{Delegation by the seller and the buyer.}

In this subsection, we allow the buyer to also hire an agent. The analysis helps us address whether it is the act of delegation itself that confers advantage to the seller or it is the separation of control of decision-making from the control of information.

Consider a two-stage game in which the seller and the buyer simultaneously hire their own agents and offer their agent a non-linear contract. This gives both the buyer and the seller the opportunity to create a commitment effect. The seller, still the only privately informed party, also announces $\kappa$. In the second-stage, the two agents bargain over quantity and price with the buyer's agent maintaining all the bargaining power. Profit for the buyer agent is defined by $a^{b}(q, \kappa)=T^{b}(q, \kappa)-T(q, \kappa)$ and profit for the seller agent is $a^{s}(q, \kappa ; \theta)=T(q, \kappa)-T^{s}(q, \kappa ; \theta)$ where $T^{b}(\cdot, \cdot)$ and $T^{s}(\cdot, ; \theta)$ are the buyer and seller contracts to their respective agents and $T(\cdot, \cdot)$ is the payment from the buyer agent to the seller agent. We maintain the convention that the seller-agent's contract is a payment to the seller and we adopt the convention that the buyer-agent's contract is a payment from the buyer. Profit for the buyer and the seller are still defined as in (9) and (10).

Given $T(\cdot, \cdot)$ and $T^{s}(\cdot, ; \theta \theta)$, the seller agent will choose $q^{a}(\kappa)$ such that $T_{q}\left(q^{a}, \kappa\right)=T_{q}^{s}\left(q^{a}, \kappa ; \theta\right)$. With the buyer's agent having all the bargaining power, any second-stage equilibrium must imply $a^{s}\left(q^{a}, \kappa ; \theta\right)=0$. This means the buyer agent will choose $T(\cdot, \cdot)$ to maximize $T^{b}-T^{s}$ by inducing the seller agent to choose $q^{a}(\cdot)$ such that $T_{q}^{b}\left(q^{a}, \kappa\right)=T_{q}^{s}\left(q^{a}, \kappa ; \theta\right)$. In the first stage, the seller's best response will imply $T^{b}\left(q^{a}, \kappa\right)=T\left(q^{a}, \kappa\right)$. The seller will choose $\kappa(\cdot)$ and $T^{s}(\cdot, \cdot)$ to maximize $T^{b}-c$. Thus, the seller's best response must satisfy $T_{q}^{b}\left(q^{a}, \mathrm{\kappa}\right)=c_{q}\left(q^{a}, \theta\right)$ as in (15). Similarly, the buyer's best response will imply $T^{b}\left(q^{a}, \kappa\right)=T^{s}\left(q^{a}, \kappa ; \theta\right)$ and $T_{q}^{s}\left(q^{a}, \kappa ; \theta\right)=b^{\prime}\left(q^{a}\right)$ as in (18). Combining these last three first-order conditions again requires in equilibrium that $q^{a}(\kappa(\theta))=q^{F}(\theta)$. The equilibrium properties implied by 
the contracts $T^{b}(\cdot, \cdot)$ and $T^{s}(\cdot, ; \theta)$, especially the seller profit property $\Sigma^{\prime}(\theta)=w_{\theta}\left(q^{F}(\theta), \theta\right)$, are identical to those in Section 3.2. This means that Proposition 2 applies to this setting as well.

If the buyer can hire an agent first, then all the analysis in the previous paragraph remains the same except for the buyer's best-response. The buyer's problem implied by solving the agent-to-agent bargaining stage and by the seller's best-response is identical to the buyer's problem under direct bargaining from section 2. Thus, a necessary condition for delegation to result in a first-best quantity is the ability of the seller to delegate simultaneously with the buyer or before the buyer. It is not sufficient. The next section will show that full delegation supports many equilibrium quantities and hence does not facilitate first-best trade.

\section{Full Delegation.}

We conclude our analysis by considering the case in which the seller delegates both the bargaining and the control of the private information to the agent. We model this case by modifying the first stage of the game so that the seller offers the agent a contract, $T^{s}(q, \kappa)$, and gives the agent a private type report, $\kappa .{ }^{14}$ In the second stage, the buyer makes the agent a take-it-or-leave-it offer. Since the buyer observes neither the seller's contract nor the seller's type report, we can model this bargaining problem as one in which the buyer offers a contract $T^{b}(q)$ to the agent. As noted in the discussion of the bargaining problem without delegation, this formulation of the second-stage is equivalent to the buyer offering the agent an incentive feasible direct contract that depends on a type report from the agent that maximizes the buyer's expected profit. By focusing on the contract that maximizes the buyer's expected profit, we keep the buyer's exogenous bargaining power the same as in the direct bargaining and partial delegation games. In the third-stage, the agent accepts or rejects the buyer's offer. If the offer is accepted, the agent chooses a quantity. We again restrict attention to differentiable contracts and solve for subgame perfect Nash equilibria. We will refer to such equilibria as "full delegation" equilibria. The structure of this game is identical to the class of games studied in the common agency literature most notably by Martimort and Stole (2002), Peters (2002, 2003), and Prat and Rustichini (2003). Consistent with this literature, we find that the set of equilibria of our full delegation game is quite large. All partial delegation equilibria continue to be equilibria but full delegation supports a wider range of equilibrium quantities. Thus, in comparison with the last section, we find that the importance of the partial delegation lies in its ability to

${ }^{14}$ Since the buyer does not observe the contract $T^{s}$ or the report $\kappa$, the buyer will have to bestrespond to a menu of contracts indexed by possible type reports. This is not a menu in the usual sense of the term as the agent is not choosing from this menu. 
implement a unique (and first-best) equilibrium quantity.

In the third stage, the agent chooses $q$ to maximize

$$
a^{f}(q, \kappa) \equiv T^{b}(q)-T^{s}(q, \kappa)
$$

subject to the agent being able to earn non-negative profit. The " $f$ " superscript is used to distinguish agent profit in this full delegation game from its profit in the partial delegation game. Let $q^{f}(\kappa)=\operatorname{argmax}_{q} a^{f}(q, \kappa)$ subject to $a^{f}(q, \kappa) \geq 0$ and let $A(\kappa) \equiv a^{f}\left(q^{f}(\kappa), \kappa\right)$. With differentiable contracts, a strictly positive value of $q^{f}(\kappa)$ will satisfy

$$
T_{q}^{b}(q)=T_{q}^{s}(q, \kappa) \text { and } T_{q q}^{b}(q) \leq T_{q q}^{s}(q, \kappa) .
$$

Differentiation of the agent's payoff, $A(\kappa)$, yields incentive compatibility conditions

$$
d A(\kappa) / d \kappa=-T_{\kappa}^{s}\left(q^{f}(\kappa), \kappa\right) \text { and } T_{q \kappa}^{s}\left(q^{f}(\kappa), \kappa\right) q^{f^{\prime}}(\kappa) \leq 0 .
$$

From (23), we see that the seller's contract affects the agent's incentive compatibility conditions in two ways. The first is through the relationship between the agent's type and its net return. For instance, lowcost types must earn lower profits than high-cost types if the seller chooses to extract significantly more rents from low-cost types (i.e., $\left.T_{\kappa}^{s}\left(q^{f}(\kappa), \kappa\right)<0\right)$. The second effect is through the marginal valuation of $q$. If $T_{q \kappa}^{s}>0$, then a high cost seller charges the agent a higher marginal price and $q^{f^{\prime}}(\kappa) \leq 0$ is required for implementation. However, if $T_{q \mathrm{\kappa}}^{s}<0$, the quantity schedule must be non-decreasing. Since the seller's contract is not observable by the buyer, the first and second stages are formally simultaneous. Thus, we need to look for a subgame perfect equilibrium in $\left(T^{s}(q, \kappa), \kappa(\theta)\right)$ and $T^{b}(q)$ given (23).

We begin with the seller's best-response problem. As in (13), the seller's profit is

$$
s\left(q^{f}(\kappa), \kappa, \theta\right)=T^{s}\left(q^{f}(\kappa), \kappa\right)-c\left(q^{f}(\kappa), \theta\right) .
$$

Given $T^{b}(q)$, the seller's problem is to choose $T^{s}(q, \kappa)$ and $\kappa(\theta)$ to induce a schedule $q^{s}(\theta)=q^{f}(\kappa(\theta))$ that maximizes (24) subject to $\mathrm{T}_{q \kappa}^{s}\left(q^{s}(\theta), \kappa(\theta)\right) q^{f^{\prime}}(\kappa(\theta)) \leq 0$ and $A(\kappa(\theta)) \geq 0$. The seller will not need to leave the agent with any information rent because the seller sets the agent's type, $\kappa . A(\kappa) \equiv 0$ means the seller's problem is equivalent to maximizing $T^{b}\left(q^{s}(\theta)\right)-c\left(q^{s}(\theta), \theta\right)$ subject to $q^{s}(\theta)=q^{f(\kappa(\theta))}$ and $T_{q \kappa}^{s}\left(q^{s}(\theta), \kappa(\theta)\right) q^{s^{\prime}}(\theta) \leq 0$. Indirect seller profit from $q^{s}(\theta)$ is

$$
s\left(q^{s}(\theta), \kappa(\theta), \theta\right) \equiv \Sigma^{f}(\theta) \equiv \Sigma^{f}(\bar{\theta})+\int_{t=\theta}^{\bar{\theta}} c_{\theta}\left(q^{s}(t), t\right) d t
$$

which implies $T^{s}\left(q^{s}(\theta), \kappa(\theta)\right)=\Sigma^{f}(\theta)+c\left(q^{s}(\theta), \theta\right)$. This seller payment is compatible with zero agent profit for all $\theta$ only if $q^{s}(\theta)$ is non-increasing in which case it can be implemented with the seller contract $T^{s}(q, \kappa)=\Sigma^{f}(\kappa)+c(q, \kappa)$. Thus, if the unconstrained optimum of $T^{b}(q)-c(q, \theta)$ is strictly positive and strictly decreasing, then the seller's best-response will induce the agent to choose $q^{s}(\theta)$ for all $\theta$ such that

$$
T_{q}^{b}\left(q^{s}(\theta)\right)=c_{q}\left(q^{s}(\theta), \theta\right)
$$

and such that agent profit is zero. That is, the seller's contract will offset any quantity distortion caused 
by the buyer's contract by setting $T_{q}^{s}(q, \kappa)$ to solve (25). Eq. (25) differs from (15) in that the buyer's contract cannot depend directly on $\kappa$ and so neither does (25).

Based on our analysis of the seller's problem, we wish to focus on equilibria for which $\kappa(\theta)$ is strictly increasing and hence strategically equivalent to truthtelling and for which the equilibrium quantity is strictly decreasing. These restrictions can only serve to limit the number of equilibria that we derive. Nonetheless, we will show that the set of equilibria satisfying these restrictions is quite large. Thus, without further loss of generality we assume $\kappa(\theta) \equiv \theta$ and $q^{\prime}(\theta)<0$.

Turning to the buyer's problem, take as given $T^{s}(q, \kappa), \kappa(\theta) \equiv \theta$, and $q^{s}(\theta)$ such that $T_{q \kappa}^{s}\left(q^{s}(\theta), \theta\right) q^{s^{\prime}}(\theta) \leq 0$. The buyer must choose $T^{b}(q)$ to induce $q^{b}(\theta)=q^{f}(\kappa(\theta))$ which maximizes $\mathscr{E}_{\theta}\left[b\left(q^{b}(\theta)\right)-T^{b}\left(q^{b}(\theta)\right)\right]$ subject to (23) and $A(\theta) \geq 0$. Using (21) and (23), the buyer's objective is equivalent to $\mathscr{E}_{\theta}\left[b\left(q^{b}(\theta)\right)-T^{s}\left(q^{b}(\theta), \theta\right)-A(\theta)\right]$ where

$$
A(\theta)=A(\bar{\theta})+\int_{t=\theta}^{\bar{\theta}} T_{\kappa}^{s}\left(q^{b}(t), t\right) d t
$$

Eq. (26) highlights the key difference between partial delegation and full delegation. With full delegation the rent extraction incentives of the agent, as captured by $A(\theta)$, are linked with the contract offered by the seller. Thus, the seller cannot provide the agent with efficient output incentives that do not provide the agent with a rent extraction motive as well. With partial delegation, this link was absent.

The solution to the buyer's best-response is derived in the appendix. Informally, the buyer's bestresponse reflects the buyer's desire to limit the information rents earned by the agent offset by a commitment effect created by the seller's contract to the agent. In the absence of a seller contract, the buyer would seek to implement the bargaining solution from Proposition 1 when $\gamma=1$. The seller's contract limits the ability of the buyer to reduce the quantity traded and thus reduce the agent's information rents by making the agent profit from low quantities negative. By varying its contract, the seller can adjust the quantity level below which agent profit is negative.

In equilibrium, $q^{b}(\theta)$ must equal $q^{s}(\theta)$. Proposition 4 reveals that the commitment effect given unobservable contracts supports economically distinct multiple equilibria by inducing distinct quantities at which the agent's zero profit constraint binds in the buyer's best-response problem. The proof provided in the appendix is constructive and uses very simple contracts to support a wide range of equilibrium quantity schedules. In particular, to support a quantity schedule $q^{*}(\theta)$ contracts of the following form will suffice:

$$
T^{s}(q, \kappa)=\Sigma^{*}(\kappa)+c(q, \kappa)
$$


and

$$
T^{b}(q)=\Sigma^{*}(\bar{\theta})+\int_{t=q^{*^{-1}}(q)}^{\bar{\theta}} c_{\theta}\left(q^{*}(t), t\right) d t+c\left(q, q^{*^{-1}}(q)\right)
$$

where $\Sigma^{*}(\kappa)=\Sigma^{*}(\bar{\theta})+\int_{t=\kappa}^{\bar{\theta}} c_{\theta}\left(q^{*}(t), t\right) d t$. This construction ensures that $T_{\kappa}^{s}(q, \kappa)=0$ at $q^{*}(\kappa)$, which from (23) is necessary for the agent to be driven to zero profits for all type reports.

Proposition 4. Let $q^{*}(\theta)$ be a strictly decreasing, differentiable function satisfying

$$
w_{q}\left(q^{*}, \theta\right)=\frac{\left(F(\theta)-\beta^{*}(\theta)\right) c_{\theta q}\left(q^{*}, \theta\right)}{f(\theta)}
$$

where $\beta^{*}(\theta)$ is a non-decreasing function such that $0 \leq \beta^{*}(\theta) \leq 1$ and let

$$
\Sigma^{*}(\bar{\theta}) \in\left[0, \int_{\theta=\underline{\theta}}^{\bar{\theta}}\left(w\left(q^{*}(\theta), \theta\right) f(\theta)+w_{\theta}\left(q^{*}(\theta), \theta\right) F(\theta)\right) d \theta\right]
$$

Then (27), $\kappa(\theta) \equiv \theta$, and (28) define a differentiable, full-delegation equilibrium in non-linear contracts in which $q^{*}(\theta)$ is traded.

Proposition 4 identifies a wide range of equilibria that are consistent with full delegation equilibria. For a given quantity schedule $q^{*}(\theta)$ satisfying (29), (30) identifies a continuum of equilibria in which the payoff to the seller differs only by a constant. This is analogous to the multiplicity that arises in the partial delegation game when seller contracts are not observable. A second source of multiplicity is the large range of equilibrium quantity schedules. This type of multiplicity did not arise in the partial delegation game, where only the efficient quantity schedule was supportable as an equilibrium. These additional equilibrium quantity schedules arise because, under full delegation, the buyer cannot condition its quantity schedule on the agent's type. As a result, there will be equilibria in which the buyer offers a contract with $T_{q}^{b}(q) \neq b^{\prime}(q)$ in order to extract information rents from the agent. The choice of $\Sigma^{*}(\kappa)$ in the seller's contract determines how large this wedge between $T_{q}^{b}(q)$ and $b^{\prime}(q)$ can be in a given equilibrium.

How large is the set of set of quantity schedules that can be supported as equilibria in the full delegation game? It should be noted that Proposition 4 provides only a partial characterization of equilibria because we have restricted attention to equilibria with contracts of the form defined by (29) and (30). However, this set of equilibria includes all incentive efficient allocations with strictly decreasing quantity schedules associated with the direct bargaining problem in section 2. It also includes an open set of incentive inefficient allocations.

Proposition 5. The set of full-delegation equilibrium allocations includes all incentive-efficient, direct- 
bargaining allocations with strictly decreasing quantity schedules.

The proof of Proposition 5 shows that the function $\beta(\theta)$ is proportional to the unconditional average welfare weight put on seller profit. Each feasible choice of type-specific seller welfare weights is shown to correspond to a feasible $\beta(\cdot)$ function. A simple example in which the full-delegation equilibrium is not incentive efficient arises when $\beta(\theta) \equiv 1$. By (29), the quantity traded when $\theta=\underline{\theta}$ is greater than the first-best level. Yet the derivation of incentive efficient allocations in the appendix shows that the quantity traded when $\theta=\underline{\theta}$ must be first best.

Therefore, the advantage of partial delegation over full delegation is that partial delegation permits an informed principal to refine the set of full delegation equilibrium outcomes that maximizes total surplus and increases seller profit for all $\theta<\bar{\theta}$ relative to direct bargaining. Since private communication between the seller and the agent cannot be observed by the buyer, it is also possible that a seller might wish to avail itself of private and public reports. In this case, a result similar to Proposition 5 would arise. ${ }^{15}$ However, the description of equilibria must now include buyer beliefs about the probability that each seller type makes a private report to the agent. Starting from a pure public reporting equilibrium, if a seller type can improve its utility through private communication, it can do so just as well by offering a different non-linear contract and not resorting to private communication. If such an opportunity existed, the initial strategy profile could not have been a pure public reporting equilibrium. As a result, there will exist an equilibrium of the game with the potential for public and private reports in which the buyer places zero probability on any seller type making private reports.

Finally, Laffont and Martimort (1998) point out that collusion between the agent and the buyer might undermine the seller's effort to increase its rents. This possibility is absent in our partial delegation and full delegation games. Any rents the agent might earn from a side contract with the buyer cannot be retained in equilibrium because the seller has full information. To be effective, incentive compatibility of the side-contract would require the buyer to pay the agent information rents the seller cannot anticipate in equilibrium. ${ }^{16}$ In a model in which the agent also has some private information, some scope for collusion

\footnotetext{
${ }^{15}$ In an earlier version of this paper, we considered a partial equilibrium game in which the buyer and the seller simultaneously announce their contracts and then the seller reports $\theta$ to the agent and $\kappa$ to the buyer. Because the agent's profit depends on $\theta$ (as well as $\kappa$ ), the buyer must pay the agent an information rent. Even though this rent will be appropriated by the seller, it will introduce the same range of equilibrium quantity distortions as in Propositions 4 and 5.

${ }^{16}$ The way in which Laffont and Martimort (1998) introduce communication limits does not have
} 
may arise. We leave this question for future research.

\section{Concluding Remarks.}

As an alternative to recent papers that focus on the potential for delegation to mitigate the impact of collusion among privately-informed agents, this paper considers the potential for delegation by an informed party in influencing bargaining outcomes. We believe our paper offers several new results. Both our partial delegation and full delegation games extend the literature on delegated bargaining by incorporating the effects of persistent private principal information. With full delegation the commitment effect induced by the introduction of a delegate can, but need not, allow an informed seller to induce favorable rent shifting relative to the case of direct bargaining. With partial delegation, our analysis shows that an informed seller can guarantee higher profit except when it is of the worst type. Thus, with a privately informed principal in a bargaining problem, it is not simply the act of delegation that confers strategic advantage to an informed party. Rather it is delegation wherein the informed party retains control of its private information that creates strong incentives for the buyer and the seller to trade the first-best quantity. To the best of our knowledge, this is the first paper to address the issue of how the control of private information influences the returns to delegation received by an informed principal. Moreover, our results show that the benefits of partial delegation to the informed party are robust to the observability of the delegation contracts and to delegation by the uninformed party as long as the uninformed party does not or cannot delegate first.

We believe our specific results are also related to several important strands of the literature. First, we believe our results add to our understanding of the economic implications of vertical integration, specifically Bork's Thesis (1978). Bork's Thesis is the term used by Bernheim and Whinston (1998) and Prat and Rustichini (2003) to describe Judge Bork's argument that vertical relationships, while viewed as anti-competitive, can facilitate efficient production. While Gal-Or (1991) and Martimort (1996) look at the role of vertical integration with a privately informed agent, our work focuses on an environment with a privately informed principal. Thus, our work can be interpreted as extending the support for Bork's Thesis as found in Bernheim and Whinston (1998) and Prat and Rustichini (2003) in a direction we believe has not yet been addressed. Again, in the context of this application, we find our results on partial delegation intriguing in light of the conventional wisdom that private information induces inefficiencies due to information rents. Since our model is not a direct extension of the work of Fershtman and Judd

any effect in our model. Thus, our argument is equivalent to their Theorem 1 asserting no equilibrium loss from side-contracting. 
(1987), Katz (1991), and Fershtman and Kalai (1997), an interesting extension would be to apply this analysis to oligopoly models.

Second, our motivation for focusing on the structure and role of delegation is related to Spulber's (1999, 2002) arguments on the importance of paying attention to the microstructure of markets in which bilateral agreements arise. For example, he demonstrates how the separation intermediation creates between investment and pricing decisions in business-to-business transactions can ameliorate hold-up problems and leads to more efficient investment. Partial delegation creates a similar separation; only now it is between trade/production and rent-seeking.

Third, from a game-theoretic perspective, the full-delegation and partial-delegation games we solve have the technical structure of common agency games with private principal information as opposed to private agent information. ${ }^{17}$ Thus, our work can also be seen as complementing the analysis of informed principal problems such as Myerson (1983) and Maskin and Tirole (1990) to include competition, via the agent, with another principal. ${ }^{18}$ Unlike in these classic papers, the choice of a contract by the informed seller influences competition between the buyer and the agent. Because the buyer and the agent bargain after the agent receives the seller's contract and the seller takes no subsequent action, agent beliefs about the seller's type do not play a role in deriving equilibria in this paper. Similarly, buyer beliefs about the seller's type do not play a role in our analysis because the buyer is constrained by the participation constraint of the agent. Buyer beliefs could play a role if the seller chose to delegate for some types and not for others. The seller has no incentive to bargain directly in this paper because doing so would only improve the capability of the buyer to minimize the rents the seller earns.

${ }^{17}$ Common agency games with a privately informed agent and symmetrically uninformed principals have been studied settings by Laffont and Tirole (1991), Martimort (1992), and Stole (1993), Bond and Gresik (1996), Mezzetti (1997), Epstein and Peters (1999), Martimort and Stole (2002), and Peters (2002, 2003). Baron (1985) studies a regulation problem with asymmetrically uninformed principals in a hierarchical environment, e.g. the EPA and price-control boards. Neither principal has private information in his model.

${ }^{18}$ More recently, Segal and Whinston (2003) use menus of contracts (in which the agent chooses a contract from the menu) to capture competition in a principal-multiple-agent model. In their model, the principal does not have any exogenously inherited private information. Bond and Gresik (1997) study a common agency problem with an informed and an uninformed principal but do not allow the uninformed principal to elicit information from the agent or the informed principal. 
Fourth, we wish to point out a possible corporate finance application. The finance literature has documented short-run excess stock price returns of $2.4 \%$ to $4.3 \%$ related to spin-off announcements. ${ }^{19}$ Numerous explanations for this phenomenon exist. The one that is closest in spirit to this paper is from Aron (1991). She argues that with a multi-division firm, share price does not track the performance of any one division very closely. Spinning off a division allows owners to provide the manager with stronger incentives based on stock price. Seward and Walsh (1996), however, do not find any evidence that the excess returns are related to stronger managerial incentives. Our results suggest an alternative explanation. In the short run, the information of the parent with respect to its interactions with the spunoff division (e.g. Ford and Visteon) remains accurate while the parent can now extract its surplus through an arm's-length relationship. Our analysis implies that by creating this arm's-length relationship, the parent eliminates the incentives faced by suppliers that in equilibrium created quantity distortions. This is inherently a short-term advantage. Over time the division's economic characteristics will likely change and its reliance on its parent for business will diminish.

Our paper suggests a number of extensions. First, in more general environments such as those in which the buyer and the seller have private information, we expect the first-best trade result and its associated benefits to be reduced but not eliminated. Second, the category management example suggests the need to include a moral hazard component to the agent's utility. In our full delegation game, renegotiation between the seller and the agent after information is shared may give the seller an additional incentive to strategically manage what it tells the agent. Once the agent learns the seller's private information, it may have an incentive to use that information to bargain for positive surplus. ${ }^{20}$ Again, one obvious way to respond to this possibility is partial delegation. However, we do not at this time rule out alternative responses to agent renegotiation in a full delegation setting as there may be important economic settings in which partial delegation is not feasible. We hope to address these and other extensions in future work.

\footnotetext{
${ }^{19}$ See Hite and Owers (1983), Miles and Rosenfeld (1983), Schipper and Smith (1983), and
} Rosenfeld (1984).

${ }^{20}$ We thank Matt Mitchell for this suggestion. 


\section{References}

Alonso, R. and N. Matouschek. 2007. Relational delegation. RAND Journal of Economics 38:1070-1089. American Names Association. 2000. What every insurance commissioner needs to know about Lloyd's. http://www.truthaboutlloyds.com/reports/whitepaperIII.htm.

Aron, D. 1991. Using the capital market as a monitor: Corporate spin-offs in an agency framework. RAND Journal of Economics 22:505-518.

Arruñada, B., L. Garicano, and L. Vásquez. 2001. Contractual allocation of decision rights and incentives: The case of automobile distribution. Journal of Law, Economics, and Organization 17:257284.

Ausubel, L. and R. Deneckere. 1989. A direct mechanism characterization of sequential bargaining with one-sided incomplete information. Journal of Economic Theory 48:18-46.

Baron, D. 1985. Non-cooperative regulation of a non-localized externality. RAND Journal of Economics 16:553-568.

Bernheim, B. and M. Whinston. 1998. Exclusive dealing. Journal of Political Economy 106:64-103. Blattberg, R. and E. Fox. 1995-6. Category Management (Guides 1 through 5). Food Marketing Institute and the Center for Retail Management, Northwestern University.

Bond, E. and T. Gresik. 1996. Regulation of multinational firms with two active governments: a common agency approach. Journal of Public Economics 59:33-53.

. 1997. Competition between asymmetrically informed principals. Economic Theory 10:227-

240.

Bork, R. H., 1978, The Antitrust Paradox: A Policy at War with Itself. Basic Books.

Cai, H and W. Cont. 2004. Agency problems in commitment and delegated bargaining. Journal of Economics and Management Strategy, 13, 703-29.

Caillaud, B. and B. Hermalin. 1993. The use of an agent in a signalling model. Journal of Economic Theory 60:83-113.

Epstein, L. and M. Peters. 1999. A revelation principle for competing mechanisms. Journal of Economic Theory 88:119-161.

The Evening Standard. June 6, 2006. Equitas increases asbestos liabilities total to Pounds 2.2 bn. London. Fershtman, C. and K. Judd. 1987. Equilibrium incentives in oligopoly. American Economic Review 77:927-940.

Fershtman, C. and E. Kalai. 1997. Unobserved delegation. International Economic Review 38:763-774. Gal-Or, Esther. 1991. A common agency with incomplete information. RAND Journal of 
Economics 22:274-286.

Fidler, S. 2004. Will Equitas come back to haunt the big companies. Financial Times November 9, 2004, London.

Gresik, T. and D. Nelson. 1994. Incentive compatible regulation of a foreign-owned subsidiary. Journal of International Economics 36:309-331.

Hall, Robert M. 2002. Direct actions and Equitas. XII Mealey's Reinsurance Report (23). http://www.robertmhall.com/articles/Equitas\&Direct.htm

Hite, G. and J. Owers. 1983. Security price reactions around corporate spin-off announcements. Journal of Financial Economics 12:409-436.

Holmström, B. and R. Myerson. 1983. Efficient and durable decision rules with incomplete information. Econometrica 51:1799-1820.

Horizon Digital Finance. 2009. myAutoloan.com. http://www2.myautoloan.com/site/page/pg4665-as206-pn_Auto_Invoice_Prices.html.

Katz, M. 1991. Game-playing agents: Unobservable contracts as precommitments. RAND Journal of Economics 22:307-328.

Koçkesen, L. and E. Ok. 2004. Strategic delegation by unobservable incentive contracts. Review of Economic Studies 71:397-424.

Laffont, J-J. and D. Martimort. 1998. Collusion and delegation. RAND Journal of Economics 29:280-305. Laffont, J-J. and J. Tirole. 1991. Privatization and incentives. Journal of Law, Economics, and Organization 7:84-105.

Martimort, D. 1992. Multi-principaux avec anti-selection. Annales d'Economique iet de Statistiques 28: 138. 1996. Exclusive dealing, common agency, and multiprincipals incentive theory. RAND Journal of Economics 27:1-31

Martimort, D. and L. Stole. 2002. The revelation and delegation principles in common agency games. Econometrica 70:1659-1674.

Maskin, E. and J.Tirole. 1990. The principal-agent relationship with an informed principal, I: Private values. Econometrica 58:379-410.

Mezzetti, C. 1997. Common agency with horizontally differentiated principals. RAND Journal of Economics 28:323-345.

Miles, J. and J. Rosenfeld. 1983. The effect of voluntary spin-off announcements on shareholder wealth. Journal of Finance 38:1597-1606. 
Myerson R. 1983. Mechanism design by an informed principal. Econometrica 51: 1767-1798. 1985. Analysis of two bargaining problems with incomplete information, in Game-theoretic

Models of Bargaining, A. Roth ed. Cambridge.

Peters, M. 2002. Common agency and the revelation principle. Econometrica 69:1349-1372. 2003. Negotiation and take it or leave it in common agency. Journal of Economic Theory 111:88-109.

Prat, A. and A. Rustichini. 2003. Games played through agents. Econometrica 71:989-1026.

Rochet, J. 1985. The taxation principle and multi-time Hamilton-Jacobi equations. Journal of Mathematical Economics 14:113-128.

Rosenfeld, J. 1984. Additional evidence on the relation between divestiture announcements and shareholder wealth. Journal of Finance 39:1437-1448.

Schelling, T. 1956. An essay on bargaining. American Economic Review 46:281-306.

Schipper, K. and A. Smith. 1983. Effects of recontracting on shareholder wealth: The case of voluntary spin-offs. Journal of Financial Economics 12:437-467.

Segal, I.and M. Whinston. 2003. Robust predictions for bilateral contracting with externalities. Econometrica 71:757-792.

Seierstad, A. and K. Sydsæter. 1987. Optimal Control Theory with Economic Applications. NorthHolland.

Seward, J. and J. Walsh. 1996. The governance and control of voluntary corporate spin-offs. Strategic Management Journal 17:25-40.

Spulber, D. 1988. Bargaining and regulation with asymmetric information about demand and supply. Journal of Economic Theory 44:251-268. 1999. Market Microstructure: Intermediaries and the Theory of the Firm. Cambridge University Press. . 2002. Market microstructure and incentives to invest. Journal of Political Economy 110: 352-381.

Standard and Poor's. 2003. Standard and Poor's Rating of the Lloyd's Market. McGraw Hill (UK). http://www.londonspecialrisks.com/pdfs/Lloyds\%20S\&P.pdf.

Stole, L. 1993. Mechanism design under common agency. Mimeo, University of Chicago. Wilson, R. 1985. Incentive efficiency of double auctions. Econometrica 53:1101-1116. 


\section{Appendix}

\section{Characterization of incentive efficient allocations for the direct bargaining problem with strictly}

\section{decreasing quantity schedules. ${ }^{21}$}

To set-up the appropriate optimization problem, we first substitute (5) into (7) to get

$$
\hat{W}=(\hat{\alpha}(\bar{\theta})-\gamma) S(\bar{\theta})+\int_{\theta=\underline{\theta}}^{\bar{\theta}}\left[(\hat{\alpha}(\theta)-\gamma) w_{\theta}(q(\theta), \theta) F(\theta) / f(\theta)+\gamma w(q(\theta), \theta)\right] f(\theta) d \theta
$$

where $\hat{\alpha}(\theta)=\mathscr{C}_{\theta}[\alpha(t) \mid t \leq \theta]$ is a conditional welfare weight reflecting the cumulative welfare effects of shifting profit to or from a seller of type $\theta$ due to incentive compatibility constraints.

Second, (6) is equivalent to

$$
\int_{\theta=\underline{\theta}}^{\bar{\theta}}[w(q(\theta), \theta)-(S(\theta)-S(\bar{\theta}))] d F(\theta) \geq S(\bar{\theta}) .
$$

Using (3) and integrating the left-hand side of (A.2) by parts implies that (A.2) is equivalent to

$$
S(\bar{\theta}) \leq \int_{\underline{\theta}}^{\bar{\theta}}\left[w(q(\theta), \theta) f(\theta)+w_{\theta}(q(\theta), \theta) F(\theta)\right] d \theta .
$$

Since $S(\bar{\theta}) \geq 0$, (6) can be satisfied as long as

$$
\int_{\underline{\theta}}^{\bar{\theta}}\left[w(q(\theta), \theta) f(\theta)+w_{\theta}(q(\theta), \theta) F(\theta)\right] d \theta \geq 0 .
$$

As a result, incentive efficient bargaining allocations can be derived by choosing $q(\cdot)$ and $S(\bar{\theta})$ to maximize (A.1) subject to (A.3), $S(\bar{\theta}) \geq 0$, (A.4), and $q^{\prime}(\cdot) \leq 0$.

As is common in the literature, the third step is to solve the optimization problem without the last constraint and then verify that it is satisfied. We need to consider two cases.

Case 1: $\gamma \geq 1 / 2$. With $\gamma>1 / 2$, it is optimal to set $S(\bar{\theta})=0$ as $\hat{\alpha}(\bar{\theta})<\gamma$. If $\gamma=1 / 2$, then it is optimal to choose $S(\bar{\theta})$ from the interval defined by (31). In either case, the Lagrangian is

$$
\mathscr{L}=(\hat{\alpha}(\theta)-\gamma+\lambda)(F(\theta) / f(\theta)) w_{\theta}(q(\theta), \theta)+(\gamma+\lambda) w(q(\theta), \theta)
$$

\footnotetext{
${ }^{21}$ This section complements the analysis in Spulber (1988) which does not provide an explicit characterization of strictly decreasing, incentive efficient allocations for multi-unit bargaining problems. The focus in his paper was to describe allocations that involved pooling (constant quantities over an open set of types) and to describe conditions on welfare weights for which a first-best quantity schedule would be incentive efficient.
} 
where $\lambda \geq 0$ is the multiplier associated with (A.4). Note that (A.5) is strictly concave in $q(\cdot)$. Define the quantity schedule $q^{1}(\theta, \lambda)$ from the first-order condition with respect to $q(\cdot)$ from (A.5). Thus, $q^{1}(\theta, \lambda)$ is the solution to

$$
w_{q}(q(\theta), \theta)=\left(1-\frac{\hat{\alpha}(\theta)}{\gamma+\lambda}\right) \frac{F(\theta)}{f(\theta)} \cdot c_{\theta q}(q(\theta), \theta) .
$$

Define $\lambda^{*}$ to be the smallest value of $\lambda \geq 0$ such that (A.4) is satisfied by $q^{1}(\cdot, \lambda)$. Because the integrand in (A.4) is continuous in $q(\cdot), \lambda^{*}$ is well-defined. If $\partial q^{1}\left(\theta, \lambda^{*}\right) / \partial \theta \leq 0$, then $q^{1}\left(\cdot, \lambda^{*}\right)$ is the incentive efficient quantity schedule. Given Assumption 1, totally differentiating (A.6) implies $\partial q^{1}\left(\theta, \lambda^{*}\right) / \partial \theta \leq 0$ if

$$
\left(\left(1-\frac{\hat{\alpha}(\theta)}{\gamma+\lambda^{*}}\right) \frac{F(\theta)}{f(\theta)}\right)^{\prime}+1 \geq 0 \text {. }
$$

Case 2: $\gamma<1 / 2$. With $\theta<1 / 2, \hat{\alpha}(\bar{\theta})>\gamma$. This case is very similar to case 1 . Constraint (A.4) will bind and the quantity schedule from the new first-order condition, now denoted by $q^{2}(\cdot, \lambda)$, is the solution to

$$
w_{q}(q(\theta), \theta)=\left(1-\frac{\hat{\alpha}(\theta)}{\hat{\alpha}(\bar{\theta})+\lambda}\right) \frac{F(\theta)}{f(\theta)} \cdot c_{\theta q}(q(\theta), \theta) .
$$

Define $\lambda^{* *}$ to be the smallest value of $\lambda \geq 0$ such that (A.4) is satisfied by $q^{2}(\cdot, \lambda) \cdot q^{2}\left(\cdot, \lambda^{* *}\right)$ is the incentive efficient quantity schedule for this case if $\partial q^{2}\left(\theta, \lambda^{* *}\right) / \partial \theta \leq 0$. Given Assumption $1, \partial q^{2}\left(\theta, \lambda^{* *}\right) / \partial \theta \leq 0$ if

$$
\left(\left(1-\frac{\hat{\alpha}(\theta)}{\hat{\alpha}(\bar{\theta})+\lambda^{* *}}\right) \frac{F(\theta)}{f(\theta)}\right)^{\prime}+1 \geq 0
$$

Q.E.D.

Proof of Lemma 1. Let $T^{b}(q, \kappa)$ be a feasible buyer strategy.

The seller's best response problem is to choose $T^{s}(q, \kappa ; \theta)$ and $\kappa(\theta)$ where $\kappa:[\underline{\theta}, \bar{\theta}] \rightarrow[\underline{\theta}, \bar{\theta}]$ (or is isomorphic to such a function) to maximize

$$
T^{s}\left(q^{a}, \kappa ; \theta\right)-c\left(q^{a}, \theta\right)
$$

subject to $\quad q^{a} \in \operatorname{argmax}_{q} T^{b}(q, \kappa)-T^{s}(q, \kappa ; \theta)$ s.t. $T^{b}(q, \kappa) \geq T^{s}(q, \kappa ; \theta)$.

For each $\theta$, define $K^{b}(\theta)=\left\{\kappa \in[\underline{\theta}, \bar{\theta}] \mid \exists q>0\right.$ s.t. $\left.T^{b}(q, \kappa) \geq c(q, \theta)\right\}$ and for each $\theta$ and for each $\kappa \in K^{b}(\theta)$, define $Q^{b}(\theta, \kappa)=\left\{q>0 \mid T^{b}(q, \kappa) \geq c(q, \theta)\right\}$. For any $q^{a} \in Q^{b}(\theta, \kappa)$ such that $\kappa \in K^{b}(\theta)$ which the seller may want to implement, seller profit will be maximized by $T^{s}\left(q^{a}, \kappa ; \theta\right)=T^{b}\left(q^{a}, \kappa\right)$. To see why, suppose $T^{s}\left(q^{a}, \kappa ; \theta\right)<T^{b}\left(q^{a}, \kappa\right)$. Adding a constant amount to $T^{s}$ for each $q$ equal to $T^{b}\left(q^{a}, \kappa\right)-T^{s}\left(q^{a}, \kappa ; \theta\right)$ does not change the agent's incentive to choose $q^{a}$ but results in zero agent profit and increases the seller's profit. This establishes (14).

One can now rewrite the seller's problem as choosing $q^{a}$ and $\kappa$ to maximize 


$$
T^{b}\left(q^{a}, \kappa\right)-c\left(q^{a}, \theta\right)
$$

subject to

$$
q^{a} \in \operatorname{argmax}_{q} T^{b}(q, \kappa)-T^{s}(q, \kappa ; \theta) \text { s.t. } T^{b}(q, \kappa) \geq T^{s}(q, \kappa ; \theta) .
$$

Denote the optimal values for each $\theta$ by $q^{s}(\theta)$ and $\kappa^{s}(\theta)$.

Differentiability of the contracts with respect to $q$ implies that agent profit is maximized where $T_{q}^{b}\left(q^{s}(\theta), \kappa^{s}(\theta)\right)=T_{q}^{s}\left(q^{s}(\theta), \kappa^{s}(\theta) ; \theta\right)$. This is true even if the constraint $T^{b}(q, \kappa) \geq T^{s}(q, \kappa ; \theta)$ binds. It also implies that the optimal quantity $q^{s}(\theta)$ must satisfy one of two possible conditions:

(I) $T_{q}^{b}\left(q^{s}(\theta), \kappa^{s}(\theta)\right)=c_{q}\left(q^{s}(\theta), \kappa^{s}(\theta)\right)$ or

(II) $T_{q}^{b}\left(q^{s}(\theta), \kappa^{s}(\theta)\right) \neq c_{q}\left(q^{s}(\theta), \theta\right)$ and $T^{b}\left(q^{s}(\theta), \kappa^{s}(\theta)\right)=c\left(q^{s}(\theta), \theta\right)$.

Under Condition (II), the seller's optimal quantity implies zero seller profit and non-zero seller marginal profit. However, $T_{q}^{b}\left(q^{s}(\theta), \kappa^{s}(\theta)\right) \neq c_{q}\left(q^{s}(\theta), \theta\right)$ and differentiability imply that there exists $q$ near $q^{s}(\theta)$ such that $T^{b}\left(q, \kappa^{s}(\theta)\right)>c(q, \theta)$ which implies positive seller profit. Hence $q^{s}(\theta)$ satisfying (II) cannot be optimal. This establishes (I) and hence (15).

Finally suppose contrary to (16) that for some $\theta$ and for some $q^{\prime} \neq q^{s}(\theta)$ that $T^{s}\left(q^{\prime}, \kappa^{s}(\theta) ; \theta\right)<T^{b}\left(q^{\prime}, \kappa^{s}(\theta)\right)$. Since $T^{s}\left(q^{s}(\theta), \kappa^{s}(\theta) ; \theta\right)=T^{b}\left(q^{s}(\theta), \kappa^{s}(\theta)\right)$, the agent would strictly prefer $q^{\prime}$ to $q^{s}(\theta)$. Hence, $q^{s}(\theta) \notin \operatorname{argmax} T^{b}\left(q, \kappa^{s}(\theta)\right)-T^{s}\left(q, \kappa^{s}(\theta) ; \theta\right)$ s.t. $T^{b}\left(q, \kappa^{s}(\theta)\right) \geq T^{s}\left(q, \kappa^{s}(\theta) ; \theta\right)$. Thus, $q^{s}(\theta)$ could not be optimal for the seller.

Q.E.D.

Proof of Lemma 2. Since each seller type $\theta$ makes a distinct public announcement, the buyer's beliefs are that the agent makes decisions under the contract $T^{s}(q, \kappa(\theta))$ with probability one. As in the proof of Lemma 1 , for any $q^{a}$ the buyer might wish to implement, $T^{b}\left(q^{a}, \kappa(\theta)\right)>T^{s}\left(q^{a}, \kappa(\theta)\right)$ cannot be optimal as buyer profit can be increased by reducing $T^{b}(q, \kappa(\theta))$ for all values of $q$ by $T^{b}\left(q^{a}, \kappa(\theta)\right)-T^{s}\left(q^{a}, \kappa(\theta)\right)$ without changing the agent's incentives to choose $q^{a}$. This establishes (17).

Given (17), one can rewrite the buyer's problem as choosing $q^{a}$ to maximize

$$
b\left(q^{a}\right)-T^{s}\left(q^{a}, \kappa(\theta)\right)
$$

subject to $\quad q^{a} \in \operatorname{argmax}_{q} T^{b}(q, \kappa)-T^{s}(q, \kappa)$ s.t. $T^{b}(q, \kappa) \geq T^{s}(q, \kappa)$.

Since the buyer can infer $\theta$ from $\kappa$, denote the buyer's optimal quantity by $q^{b}(\theta)$.

Differentiability of the contracts again implies that $T_{q}^{b}\left(q^{b}(\theta), \kappa(\theta)\right)=T_{q}^{s}\left(q^{b}(\theta), \kappa(\theta)\right)$. It also implies that the optimal quantity $q^{b}(\theta)$ must satisfy one of two possible conditions:

(I) $T_{q}^{s}\left(q^{b}(\theta), \kappa(\theta)\right)=b^{\prime}\left(q^{b}(\theta)\right)$ or

(II) $T_{q}^{s}\left(q^{b}(\theta), \kappa(\theta)\right) \neq b^{\prime}\left(q^{b}(\theta)\right)$ and $T^{s}\left(q^{b}(\theta), \kappa(\theta)\right)=b\left(q^{b}(\theta)\right)$.

As in Lemma 1, the requirement that the contracts be differentiable rules out condition (II) and establishes (18).

Finally, if (19) is violated for some $q^{\prime}$ then the agent prefers $q^{\prime}$ over $q^{b}(\theta)$ which means $q^{b}(\theta)$ is 
not agent incentive compatible.

Lemma 3. Let $\left(\left(T^{s}, \mathrm{\kappa}\right), T^{b}, q^{a}\right)$ be a positive-trade, differentiable partial delegation equilibrium that results in equilibrium quantity $q^{*}(\theta)$ and that has the following properties: (a) there exists a set of types $P(\bar{\kappa})$ such that for all $\theta \in P(\bar{\kappa}), \kappa(\theta)=\bar{\kappa}$, and (b) there exists at least two types in $P(\bar{\kappa}), \theta^{\prime}$ and $\theta^{\prime \prime}$, for which there exists $q>0$ such that $T^{s}\left(q, \bar{\kappa} ; \theta^{\prime}\right) \neq T^{s}\left(q, \bar{\kappa} ; \theta^{\prime \prime}\right)$ and $b(q, \bar{\kappa}) \geq T^{s}(q, \bar{\kappa} ; \theta)$ for $\theta \in\left\{\theta^{\prime}, \theta^{\prime \prime}\right\}$. Then $b^{\prime}\left(q^{*}(\theta)\right)=T_{q}^{s}\left(q^{*}(\theta), \bar{\kappa} ; \theta\right)$ for all $\theta \in P(\bar{\kappa})$.

Proof of Lemma 3. Given pooling on $\kappa$ but contract separation, we can focus on the buyer's response when $\bar{\kappa}$ is announced. The buyer's problem is to choose $T^{b}(q, \bar{\kappa})$ to maximize

$$
\int_{\theta \in P(\bar{\kappa})}\left[b\left(q^{a}(\theta)\right)-T^{b}\left(q^{a}(\theta), \bar{\kappa}\right)\right] d F(\theta \mid P(\bar{\kappa}))
$$

subject to (i) For each $\theta \in P(\bar{\kappa})$, if $q^{a}(\theta)>0$, then

$$
q^{a}(\theta) \in \operatorname{argmax}_{q} T^{b}(q, \bar{\kappa})-T^{s}(q, \bar{\kappa} ; \theta) \text { s.t. } T^{b}(q, \bar{\kappa}) \geq T^{s}(q, \bar{\kappa} ; \theta) .
$$

For all $\theta \in P(\bar{\kappa})$ such that $q^{b}(\theta)>0$, constraint (i) can be restated as requiring

$$
T^{b}\left(q^{b}(\theta), \bar{\kappa}\right)=T^{s}\left(q^{b}(\theta), \bar{\kappa} ; \theta\right)+\max _{\mathrm{q}}\left[T^{b}(q, \bar{\kappa})-T^{s}(q, \bar{\kappa} ; \theta)\right] .
$$

Given Lemma 1, a necessary condition for a positive trade equilibrium is (16) which implies that for all $q$ and for all $\theta \in P(\bar{\kappa}), T^{b}(q, \bar{\kappa}) \leq T^{s}(q, \bar{\kappa} ; \theta)$. Thus, to be part of an positive trade equilibrium profile it must be that $T^{b}(q, \bar{\kappa}) \leq \underline{T}^{s}(q, \bar{\kappa}) \equiv \min _{\theta \in \mathrm{P}(\overline{\mathrm{\kappa}})} T^{s}(q, \overline{\mathrm{\kappa}} ; \theta)$. Remark: If the min does not exist for some seller strategy $\left(T^{s}(q, \kappa ; \theta), \kappa(\theta)\right)$, the seller strategy cannot be part of a positive trade equilibrium unless the lack of existence only occurs at quantities that are not optimal for the buyer.

Define for all $\theta \in P(\bar{\kappa}), Q(\theta)=\left\{q>0 \mid T^{s}(q, \bar{\kappa} ; \theta)=\underline{T}^{s}(q, \bar{\kappa})\right\}$. If $Q(\theta)$ is empty for some $\theta$, then either $q^{b}(\theta)=0$ or there exists some $\theta^{\prime} \in P(\bar{\kappa})$ for which $T^{b}\left(q^{b}\left(\theta^{\prime}\right), \bar{\kappa}\right)>T^{s}\left(q^{b}\left(\theta^{\prime}\right), \bar{\kappa} ; \theta^{\prime}\right)$. Both possibilities are inconsistent with a positive trade equilibrium. Thus, assume $Q(\theta)$ is non-empty for all $\theta \in P(\bar{\kappa})$. In order for $\left(T^{b}(q, \kappa),\left(T^{s}(q, \kappa ; \theta), \kappa(\theta)\right)\right)$ to constitute a positive trade equilibrium it must be that $q^{b}(\theta) \in Q(\theta)$ for all $\theta \in P(\bar{\kappa})$.

Next for all $\theta \in P(\bar{\kappa})$, define $Q^{b}(\theta)=\operatorname{argmax}_{q} b(q)-T^{s}(q, \bar{\kappa} ; \theta)$ s.t. $q \in \underline{Q}(\theta)$. $Q^{b}(\theta)$ describes all the quantities, consistent with a positive trade equilibrium, that maximize the buyer's expected profit on $P(\bar{\kappa})$. This set must be non-empty for each $\theta \in P(\bar{\kappa})$ in order for the specified profile to be a positive trade equilibrium. It must also be that $q^{b}(\theta) \in Q^{b}(\theta)$ for all $\theta \in P(\bar{\kappa})$. If not, the buyer could choose a better quantity for some $\theta \in P(\bar{\kappa})$ without affecting the agent incentive compatibility or zero profit conditions. Now assume by contradiction that there exists $\hat{\theta} \in P(\bar{\kappa})$ for which $b^{\prime}\left(q^{b}(\hat{\theta})\right) \neq T_{q}^{s}\left(q^{b}(\hat{\theta}), \bar{\kappa} ; \hat{\theta}\right)$. This means there exists no $\bar{\delta}>0$ such that, for all $\delta<\bar{\delta}$, $\left(q^{b}(\hat{\theta})-\delta, q^{b}(\hat{\theta})+\delta\right) \subseteq Q(\hat{\theta})$. Otherwise, there would exist an incentive compatible, zero agent profit quantity that would improve the buyer's profit. In other words, $q^{b}(\hat{\theta})$ must either be an isolated point or 
a boundary point of $Q(\hat{\theta})$. (An isolated point would exist if locally $T^{s}(q, \bar{\kappa} ; \hat{\theta})=\underline{T}^{s}(q, \bar{\kappa})$ at a single point.)

Because $T^{s}(q, \kappa ; \theta)$ is required to be differentiable for all $q$ and for all $\theta$, the fact that $q^{b}(\hat{\theta})$ is either an isolated point or a boundary point of $Q(\hat{\theta})$ implies that there also exists $\tilde{\theta} \in P(\bar{\kappa})$ such that $T^{s}\left(q^{b}(\hat{\theta}), \bar{\kappa} ; \hat{\theta}\right)=T^{s}\left(q^{b}(\hat{\theta}), \bar{\kappa} ; \tilde{\theta}\right)$. This fact implies that $q^{b}(\hat{\theta}) \in Q(\tilde{\theta})$ and in order for $T^{b}(q, \bar{\kappa})$ to be differentiable at $q^{b}(\hat{\theta})$ it must be that $T_{q}^{s}\left(q^{b}(\hat{\theta}), \bar{\kappa} ; \hat{\theta}\right)=T_{q}^{s}\left(q^{b}(\hat{\theta}), \bar{\kappa} ; \tilde{\theta}\right)$.

We now show that it cannot be part of the buyer's best response to implement $q^{b}(\hat{\theta})$. The fact the $q^{b}(\hat{\theta})$ is either an isolated point of $Q(\hat{\theta})$ or a boundary point of $Q(\hat{\theta})$ where the gradient $b^{\prime}\left(q^{b}(\hat{\theta})\right)-T_{q}^{s}\left(q^{b}(\theta), \bar{\kappa} ; \hat{\theta}\right)$ points out of the set and the differentiability of $T^{s}(q, \kappa ; \theta)$ imply an alternative quantity $q^{\prime}(\hat{\theta})$ satisfying the following properties exists: There exists $\bar{\eta}>0$, such that for all $\eta<\bar{\eta}$, $q^{\prime}(\hat{\theta}) \in Q^{\prime}(\hat{\theta}, \eta) \equiv\left\{q^{b}(\hat{\theta})-\eta, q^{b}(\hat{\theta})+\eta\right\}$ for which $b\left(q^{\prime}(\hat{\theta})\right)-T^{s}\left(q^{\prime}(\hat{\theta}), \bar{\kappa} ; \hat{\theta}\right)>b\left(q^{b}(\hat{\theta})\right)-T^{s}\left(q^{b}(\hat{\theta}), \bar{\kappa} ; \hat{\theta}\right)$ and $T^{s}\left(q^{\prime}(\hat{\theta}), \bar{\kappa} ; \hat{\theta}\right)>T^{s}\left(q^{\prime}(\hat{\theta}), \bar{\kappa} ; \theta\right)$ for some $\theta \in P(\bar{\kappa})$. That is, differentiability of the seller's contract implies that one can move infinitesimally away from $q^{b}(\hat{\theta})$ along $T^{s}(q, \bar{\kappa} ; \hat{\theta})$ in a direction that improves the buyer's profit. Because such a move means the new quantity is no longer in $Q(\hat{\theta}), T^{s}\left(q^{\prime}(\hat{\theta}), \bar{\kappa} ; \hat{\theta}\right)$ can no longer equal $\underline{T}^{s}\left(q^{\prime}(\hat{\theta}), \bar{\kappa}\right)$.

One way to ensure that $q^{\prime}(\hat{\theta})$ and $q^{b}(\theta)$ for $\theta \in P(\bar{\kappa}) \mid \hat{\theta}$ are agent incentive compatible is to set $T^{b}\left(q^{\prime}(\hat{\theta}), \bar{\kappa}\right)=T^{s}\left(q^{\prime}(\hat{\theta}), \bar{\kappa} ; \hat{\theta}\right)$ and to raise $T^{b}\left(q^{b}(\theta), \bar{\kappa}\right)$ for all $\theta \in P(\bar{\kappa}) \backslash \hat{\theta}$ by $T^{s}\left(q^{\prime}(\hat{\theta}), \bar{\kappa} ; \hat{\theta}\right)-T^{s}\left(q^{\prime}(\hat{\theta}), \bar{\kappa} ; \theta^{*}\right)$ where $\theta^{*}$ is a type for which $T^{s}\left(q^{\prime}(\hat{\theta}), \bar{\kappa} ; \theta^{*}\right)=\underline{T}^{s}\left(q^{\prime}(\hat{\theta}), \bar{\kappa}\right)$. For all $q \neq q^{b}(\theta)$ for some $\theta \in P(\bar{\kappa}) \backslash \hat{\theta}$, one must increase $T^{b}(q, \bar{\kappa})$ enough to preserve the differentiability of the contract without destroying the incentive compatibility of $q^{b}(\theta)$. The marginal gain to the buyer at $q^{b}(\hat{\theta})$ (i.e. $\eta=0)$ is $b^{\prime}\left(q^{b}(\hat{\theta})\right)-T^{s}\left(q^{b}(\hat{\theta}), \bar{\kappa} ; \hat{\theta}\right)>0$ while the marginal cost at $q^{b}(\hat{\theta})$ is $\int_{P(\bar{\kappa}) \mid \hat{\theta}}\left[T_{q}^{s}\left(q^{b}(\hat{\theta}), \bar{\kappa}, \hat{\theta}\right)-T_{q}^{s}\left(q^{b}(\hat{\theta}), \bar{\kappa} ; \theta\right)\right] d F(\theta \mid P(\bar{\kappa}))=0$. For $\eta$ sufficiently close to zero, the gain to switching from $q^{b}(\hat{\theta})$ to $q^{\prime}(\hat{\theta})$ must by continuity improve the buyer's expected profit on $P(\bar{\kappa})$. Thus, the fact that $b^{\prime}\left(q^{b}(\hat{\theta})\right) \neq T_{q}^{s}\left(q^{b}(\theta), \bar{\kappa} ; \hat{\theta}\right)$ implies that inducing $q^{b}(\theta)$ on $P(\bar{\kappa})$ is not part of a best response for the buyer.

Q.E.D.

Construction of Non-Linear Contracts for Section 3.2: The non-linear contracts that support $q^{F}(\theta)$ as an equilibrium quantity are not unique for reasons described in section 3.2. However, one pair of contracts is sufficient to prove Proposition 2. Assume the seller's contract is of the form

$$
T^{s}(q, \kappa ; \theta)=b(q)-\phi+\int_{t=q}^{q^{F}(\kappa)}\left[\left(q^{F}\right)^{-1}(t)-\kappa\right] d t,
$$

and assume the buyer's contract is of the form

$$
T^{b}(q, \kappa)=b(q)-\phi .
$$

Seller contract (A.10) implies $b(q)-T^{s}(q, \kappa ; \theta)$ is strictly concave in $q$ for all $\kappa$ and $q^{b}(\kappa)=q^{F}(\kappa)$ as long 
as buyer profit is non-negative at this quantity. This can be guaranteed by $\phi \geq 0$. Therefore, (A.11) is a best-response for the buyer.

Similarly given (A.11), the seller will implement $q^{F}(\theta)$ if $\phi \leq b\left(q^{F}(\bar{\theta})\right)-c\left(q^{F}(\bar{\theta}), \bar{\theta}\right)$. Given (A.10) - (A.11), agent profit is strictly concave in $q$ for all $\kappa$ (since $\left.T_{q q}^{b}-T_{q q}^{s}=\left(q^{F^{-1}}\right)^{\prime}(q)<0\right)$ and maximized at $q^{a}(\kappa ; \theta)=q^{F}(\kappa)$. Thus, (A.10) implies $s\left(q^{F}(\kappa), \kappa, \theta\right)=b\left(q^{F}(\kappa)\right)-c\left(q^{F}(\kappa), \theta\right)-\phi$ so seller profit is maximized at $\kappa=\theta$. Since the seller's strategy defined by (A.10) and $\kappa(\theta)=\theta$ implements the seller's optimal quantity, it is a best-response for the seller to buyer strategy (A.11). Therefore, (A.10), $\kappa(\theta)=\theta$, and (A.11) are equilibrium strategies.

\section{The buyer's best-response in the full-delegation game.}

The buyer's best-response to any seller strategy solves

$$
\begin{gathered}
\max _{\mathrm{q}(\cdot)} \mathscr{E}_{\theta}\left[b(q(\theta))-T^{s}(q(\theta), \theta)-A(\theta)\right] \\
\text { s.t. } a) A^{\prime}(\theta)=-T_{\kappa}^{s}(q(\theta), \theta) \\
\text { b) } A(\theta) \geq 0 \\
\text { c) } T_{q \kappa}^{s}(q(\theta), \theta) q^{\prime}(\theta) \leq 0 .
\end{gathered}
$$

In contrast to the standard principal agent problem in which (A.12b) binds only for the least cost type, the solution to the seller's problem dictates that in equilibrium $A(\theta)$ will equal zero for all $\theta$ and thus can bind on open sets of types. Thus, rather than characterizing the buyer's best-response to any seller strategy, we instead seek to derive necessary and sufficient conditions for which the buyer's best-response implies $A(\theta) \equiv 0$. To do this we formulate the supplier's problem as an optimal control problem with a pure state constraint. Following Seierstad and Sydsaeter (1987), we form the Hamiltonian

$$
\mathcal{H}(q, A, \mu, \theta)=\left(b(q(\theta))-T^{s}(q(\theta), \theta)-A(\theta)\right) f(\theta)-\mu(\theta) T_{\kappa}^{s}(q(\theta), \theta)
$$

and the associated Lagrangian

$$
\mathscr{L}(q, A, \mu, \lambda, \theta)=\mathcal{H}(q, A, \mu, \theta)+\lambda(\theta) A(\theta) .
$$

Theorem 5.1 in Seierstad and Sydsaeter (1987) shows that $\left(q^{*}(\theta), A(\theta)=0\right)$ maximizes (A.12) if there exists a piecewise continuous function $\lambda(\theta) \geq 0$ and a continuous and piecewise continuously differentiable function $\mu(\theta)$ satisfying the following conditions:

$$
\begin{aligned}
& \text { (a) } q^{*}(\theta) \in \operatorname{argmax}_{q} \mathcal{H}(q, A, \mu, \theta), \\
& \text { (b) } \dot{\mu}(\theta)=f(\theta)-\lambda(\theta) \text {, and } \\
& (c) \mu(\underline{\theta}) \leq 0 \text { and } \mu(\bar{\theta}) \geq 0 .
\end{aligned}
$$

When (A.13) is concave in $q$, condition (A.14a) is equivalent to 


$$
\left(b_{q}\left(q^{*}(\theta)\right)-T_{q}^{s}\left(q^{*}(\theta), \theta\right)\right) f(\theta)=\mu(\theta) \cdot T_{q \mathrm{\kappa}}^{s}\left(q^{*}(\theta), \theta\right)
$$

The contracts we use to construct equilibria in the proof of Proposition 4 satisfy this concavity requirement so $q^{*}(\theta)$ differs from the buyer's unconstrained first best if $\mu(\theta) \neq 0$. In such a case, the buyer finds it optimal to distort the quantity schedule in order to reduce the agent's information rents.

Define the non-decreasing function,

$$
\beta(\theta)=\beta(\underline{\theta})+\int_{t=\underline{\theta}}^{\theta} \lambda(t) d t .
$$

By incentive compatability constraint (A.12a), if the buyer wishes to change agent $\theta$ 's profit by one dollar, it must also adjust the profit for all lower type agents by one dollar. Thus, $\beta(\theta)$ is the aggregate shadow price associated with the non-negative profit constraint, (A.12b). Condition (A.14b) implies that $\mu(\theta)=F(\theta)-\beta(\theta)+k$ where $k$ is a constant. Without loss of generality, we can set $k$ equal to 0 . Because $\beta(\cdot)$ is non-decreasing, (A.14c) implies that $0 \leq \beta(\theta) \leq 1$ for all $\theta$. Note that higher values of $\beta(\cdot)$ reduce the buyer's incentive to limit the agent's marginal information rents by trading a lower quantity (via $\mu(\cdot))$. Therefore, if $T^{s}(q, \kappa)$ implies that (A.13) is concave in $q$, then $\left(q^{*}(\theta), A(\theta) \equiv 0\right)$ maximizes (A.12) only if there exists a non-decreasing function $\beta(\theta)$ such that, for all $\theta, 0 \leq \beta(\theta) \leq 1$, and

$$
b_{q}\left(q^{*}(\theta)\right)-T_{q}^{s}\left(q^{*}(\theta), \theta\right)=(F(\theta)-\beta(\theta)) T_{q \kappa}^{s}\left(q^{*}(\theta), \theta\right) / f(\theta) .
$$

Furthermore, because the Hamiltonian and the state constraint, $A(\theta) \geq 0$, are linear in the state variable, $A(\cdot)$, Theorem 3 in chapter 5 of Seierstad and Sydsæter (1987) implies that these conditions are also sufficient. Finally, the buyer can always refuse to offer the agent a contract and earn zero profit. The buyer will do so only if its expected profit is negative. Thus, implementing $\left(q^{*}(\theta), A(\theta) \equiv 0\right)$ is a best response for the buyer to any seller strategy $\left(T^{s}, \kappa(\theta) \equiv \theta\right)$ for which (A.12) is concave in $q$ if, and only if, $\beta(\cdot)$ is non-decreasing, $0 \leq \beta(\cdot) \leq 1$, and (A.21) are satisfied and $\mathscr{E}_{\theta}\left[b\left(q^{*}(\theta)\right)-T^{s}\left(q^{*}(\theta), \theta\right)\right] \geq 0$.

Proof of Proposition 4. Consider a candidate equilibrium quantity schedule, $q^{*}(\theta)$, that is strictly decreasing. We begin by showing that using (27) to induce $q^{*}(\theta)$ with $\kappa(\theta)=\theta$ is a best-response for the seller to the buyer contract, (28). As previously discussed, the seller's objective, $T^{b}(q)-c(q, \theta)$, is independent of $\kappa$. This observation gives us great latitude in choosing $\kappa(\theta)$. Eq. (27) also implies that the derivative of the seller's objective with respect to $q$ (equivalent to (25)) is

$$
c_{q}\left(q, q^{*^{-1}}(q)\right)-c_{q}(q, \theta)
$$

Because $c_{q \theta}(q, \theta)>0$, (A.17) is strictly positive for $q<q^{*}(\theta)$ and strictly negative for $q>q^{*}(\theta)$. Thus, (24) is maximized at $q^{*}(\theta)$. Moreover, at $q=q^{*}(\theta)$, (27) implies zero agent profit and $T_{q}^{b}(q)=T_{q}^{s}(q, \theta)$. The assumption $c_{q \theta}>0$ ensures that agent profit is also maximized at $q^{*}(\theta)$. To implement $q^{*}(\theta), \kappa(\theta)$ must be strictly monotone. Without loss of generality, we can set $\kappa(\theta) \equiv \theta$. Consequently, $T^{s}(\cdot, \cdot)$ defined 
by (27) and $\kappa(\theta) \equiv \theta$ is a best-response to (28) as long as $\Sigma^{*}(\theta)$ is non-negative (to guarantee non-negative seller profit for all $\theta$ ).

Next we need to determine if using (28) to induce $q^{*}(\theta)$ is a best-response for the buyer to the seller strategy of (27) and $\kappa(\theta) \equiv \theta$. First note that (28) makes the buyer's Hamiltonian, (A.13), strictly concave in $q$. Then note that substituting (27) into (A.16) implies that it is optimal for the buyer to offer a contract that implements $q^{*}(\theta)$ and results in zero agent profit if, and only if, there exists a non-decreasing function $\beta(\theta)$ that maps into $[0,1]$ such that

$$
b_{q}\left(q^{*}(\theta)\right)-c_{q}\left(q^{*}(\theta), \theta\right)=(F(\theta)-\beta(\theta)) c_{q \theta}\left(q^{*}(\theta), \theta\right) / f(\theta) .
$$

Since the left-hand side of (A.18) is $w_{q}\left(q^{*}(\theta), \theta\right)$, (A.18) is equivalent to (29). Expected equilibrium buyer profit will be non-negative if

$$
\Sigma^{*}(\bar{\theta}) \leq \int_{\theta=\underline{\theta}}^{\bar{\theta}}\left(w\left(q^{*}(\theta), \theta\right) f(\theta)+w_{\theta}\left(q^{*}(\theta), \theta\right) F(\theta)\right) d \theta
$$

which is the upper bound in (30).

Q.E.D.

Proof of Proposition 5. The set of strictly decreasing, incentive efficient quantity schedules, defined by (A.6) and (A.8), vary based on the conditional welfare weights, $\hat{\alpha}(\theta)=\mathscr{E}_{\theta}[\alpha(t) \mid t \leq \theta]$ and a Lagrange multiplier $\left(\lambda^{*}\right.$ or $\left.\lambda^{* *}\right)$. First, fix the welfare weights $\alpha(\theta)$ and $\gamma$ such that $\gamma \geq 1 / 2$ and such that (A.6) defines a strictly decreasing quantity schedule. This quantity schedule is a full-delegation equilibrium quantity for $\beta(c)=\hat{\alpha}(c) F(c) /\left(\gamma+\lambda^{*}\right)$. Second, fix the welfare weights $\alpha(\theta)$ and $\gamma$ such that $\gamma<1 / 2$ and such that (A.8) defines a strictly decreasing quantity schedule. This schedule is a full-delegation equilibrium schedule for $\beta(c)=\hat{\alpha}(c) F(c) /\left(\hat{\alpha}(\bar{c})+\lambda^{* *}\right)$. Simple inspection of both cases reveals that the $\beta(\cdot)$ functions are non-negative, non-decreasing, and always less than or equal to one. 